\title{
Ascomicetos de la Reserva de la Biosfera Isla Cozumel, México
}

\section{Ascomicetes of the Cozumel Island Biosphere Reserve, Mexico}

\section{Acta Botanica Mexicana}

\author{
Tania Raymundo' (ID, Michelle Martínez-Pinedal (D), Pamela E. Reyes³ (iD, Aurora Cobos-Villagrán³ (iD, Yenitze A. García- \\ Martínez ${ }^{3}$ (D), Adrián A. Tun² (iD, Ricardo Valenzuela1,4 (D)
}

\section{Resumen:}

Antecedentes y Objetivos: Los ascomicetos son el grupo más diverso del reino Fungi; han sido descritos como saprobios, necrótrofos, biótrofos y endófitos. En la Isla de Cozumel, Quintana Roo, México, aunque recientemente se han citado 29 especies, no existe un estudio exclusivo de este grupo de hongos. El objetivo del presente estudio es hacer una lista de las especies de ascomicetos de tres Áreas Naturales Protegidas (ANPs) de la Reserva de la Biosfera Isla Cozumel, México.

Métodos: Se realizaron cuatro exploraciones en las Áreas Naturales Protegidas de la Reserva de la Biosfera Isla Cozumel: Centro de Conservación y Educación Ambiental, Parque Ecológico Punta Sur y Zona Arqueológica de San Gervasio Chen-tuk, todas administradas por la Fundación Parques y Museos de Cozumel. Los ejemplares recolectados fueron estudiados, descritos y determinados de acuerdo con las técnicas tradicionales en micología. El material está depositado en el Herbario ENCB.

Resultados clave: Se determinaron 40 especies de ascomicetos; de éstos, 15 se citan por primera vez para Quintana Roo y ocho se registran para la Isla de Cozumel. Bertia tropicalis se cita por primera vez para México y Platystomum mexicanum se describe como especie nueva para la ciencia. Conclusiones: La clase Sordariomycetes es la mejor estudiada con 28 especies, mientras que el orden Xylariales, con 25, tiene 62.5\% del total de las 40 registradas. La familia Hypoxylaceae, con $40 \%$ de los taxa determinados, es la familia más representada. Hypoxylon lenormandii, Rhytidhysteron neorufulum y $R$. rufulum fueron encontradas en todas las ANPs estudiadas.

Palabras clave: Caribe mexicano, Hypoxylaceae, especie nueva, nuevo registro, Platystomum, Sordariomycetes.

\begin{abstract}
:
Background and Aims: Ascomycetes are the most diverse group of the kingdom Fungi; they have been described as saprobes, necrotrophs, biotrophs and endophytes. On the Island Cozumel, Quintana Roo, Mexico, 29 species have recently been cited; however, there is no exclusive study of this group. The objective of this study is to present a list of ascomycetes species from three Protected Natural Areas in the Cozumel Island Biosphere Reserve, Mexico.

Methods: Four explorations were carried out in the Natural Protected Areas of the Cozumel Island Biosphere Reserve: Centro de Conservación y Educación Ambiental, Parque Ecológico Punta Sur and Zona Arqueológica de San Gervasio, Chen-tuk; all are administrated by the Fundación Parques y Museos of Cozumel. The collected specimens were studied, described and determined according to the traditional mycological techniques. The material is deposited in the ENCB Herbarium.

Key results: Forty species of ascomycetes are determinated, of these 15 are cited for the first time for Quintana Roo, and eight are recorded for Cozumel Island. Bertia tropicalis is a new record from Mexico and Platystomum mexicanum is described as a species new to science.

Conclusions: The class Sordariomycetes is the best represented with 28 species, while the order Xylariales with 25 species has $62.5 \%$ of the total of 40 registered. The family Hypoxylaceae, with $40 \%$ of the determinated taxa, is the best represented family. Hypoxylon lenormandii, Rhytidhysteron neorufulum and $R$. rufulum were found in all Natural Protected Areas studied.
\end{abstract}

Key words: Mexican Caribbean, Hypoxylaceae, new species, new record, Platystomum, Sordariomycetes.

${ }^{1}$ Instituto Politécnico Nacional, Escuela Nacional de Ciencias Biológicas, Departamento de Botánica, Laboratorio de Micología, 11340 Cd. Mx., México.

${ }^{2}$ Fundación de Parques y Museos de Cozumel, Carretera transversal km 7, 77600 Cozumel, Quintana Roo, México.

${ }^{3}$ Instituto Politécnico Nacional, Escuela Nacional de Ciencias Biológicas, Posgrado en Biociencias, 11340 Cd. Mx., México.

${ }^{4}$ Autor para la correspondencia: rvalenzg@ipn.mx
Recibido: 22 de octubre de 2020.

Revisado: 11 de noviembre de 2020. Aceptado por Marie-Stéphanie Samain: 14 de abril de 2021.

Publicado Primero en línea: 06 de mayo de 2021. Publicado: Acta Botanica Mexicana 128 (2021).
Citar como:

Raymundo, T., M. Martínez-Pineda, P. E. Reyes, A. Cobos-Villagrán, Y. A. García-Martínez, A. A. Tun y R. Valenzuela. Ascomicetos de la Reserva de la Biosfera Isla Cozumel, México. Acta Botanica Mexicana 128: e1806. DOI: https://doi.org/10.21829/abm128.2021.1806 


\section{Introducción}

La Reserva de la Biosfera Isla Cozumel tiene una superficie total de $520 \mathrm{~km}^{2}$, se ubica entre los paralelos $20^{\circ} 36^{\prime}$ a $20^{\circ} 16^{\prime} \mathrm{N}$ y los meridianos $86^{\circ} 44^{\prime}$ a $87^{\circ} 20^{\prime} \mathrm{O}$ en el Caribe Mexicano, en el estado de Quintana Roo, en la costa oriental de la Península de Yucatán, México (García, 2004). Cozumel es una isla oceánica de origen coralino, que nunca estuvo conectada al continente (CONANP, 2016). Este aislamiento la convirtió en un lugar propicio para la generación de endemismos, ya que a lo largo del tiempo geológico sus poblaciones han evolucionado en forma independiente. Cuatro grupos de suelos se distribuyen en la superficie insular con extensiones muy desiguales. El principal es el Rendzina, que ocupa $86.51 \%$ de la superficie insular repartiéndose por su zona central. El segundo en cobertura es el Solonchak, que abarca $10.86 \%$ de la superficie del territorio y se distribuye principalmente en las zonas pantanosas de los extremos sur, norte y en una porción de la costa nororiental (siendo en el primer caso de tipo órtico y en los otros dos de tipo gléyico). El tercero es el suelo denominado Gleysol (de tipo mólico), el cual ocupa $1.05 \%$ de la superficie y se encuentra en la costa oriental immediatamente al norte de la carretera transversal. Finalmente, el Regosol (de tipo calcárico) ocupa $0.5 \%$ de la superficie insular y se sitúa en una estrecha franja de la costa al norte de San Miguel de Cozumel (CONANP, 2007). Sus ecosistemas son variados, pues existen arrecifes coralinos, pastos marinos, manglares, vegetación de dunas costeras, tasistales-saibales; la selva baja caducifolia y mediana subcaducifolia representan los tipos de vegetacion predominantes con 70\% (RomeroNájera, 2004). Entre las especies dominantes se tienen a Acacia pringlei Rose (huamuche), Brosimum alicastrum Sw. (ramón), Bursera simaruba (L.) Sarg. (palo mulato), Caesalpinia yucatanensis (Britton \& Rose) Greenm. (palo gusano), Cecropia obtusifolia Bertol. (guarumo), Ceiba aesculifolia (Kunth) Britten \& Baker f. (ceibillo), Coccoloba barbadensis Jacq. (uvero), Guaiacum sanctum L. (guayacán), Pithecellobium mangense (Jacq.) Macbride (cucharo) y Vitex gaumeri Greenm. (yaxnik) (CONANP, 2016).

Los ascomicetos son el grupo de hongos con mayor número de especies descritas en el Reino Fungi (Kirk et al., 2008). El grupo se caracteriza por la presencia de generalmente ocho ascosporas dentro de sacos denominados as- cas, organizadas en cuerpos fructíferos llamados ascomas o ascocarpos. La mayoría de sus especies son saprobias, necrótrofas o biótrofas parásitas de plantas o animales, incluido el hombre. Sin embargo, cada vez son más las que se encuentran como endófitas (Webster y Weber, 2007).

Los primeros trabajos que registran ascomicetos de la Reserva de la Biosfera Isla Cozumel son los de Kohlmeyer (1984) y González et al. (2001), quienes citaron 15 especies de ascomicetos marinos para las costas de la isla. Recientemente se han citado algunas especies de ascomicetos características de la selva baja caducifolia, entre ellas se encuentran Daldinia eschscholtzii (Ehrenb.) Rehm (Barbosa-Reséndiz et al., 2020), Hypoxylon cinnabarinum (Henn.) Henn., $H$. erythrostroma J.H. Mill., H. haematrostroma Mont., $H$. investiens (Schwein.) M.A. Curtis, H. jaklitschii Sir \& Kuhnert, H. lenormandii Berk. \& M.A. Curtis, H. perforatum (Schwein.) Fr., H. rickii Y.M. Ju \& J.D. Rogers, H. subgilvum Berk \& Broome (Reyes et al., 2020), Rhytidhysteron neorufulum Thambug. \& K.D. Hyde y R. rufulum (Spreng.) Speg. (Cobos-Villagrán et al., 2020). Además, García-Martínez et al. (2021b) reportaron 16 ascomicetos de las 20 especies de hongos asociadas a Rhizophora mangle L. (mangle rojo) para la reserva y García-Martínez et al. (2021a) citan a Mycoporum buckii R.C. Harris sobre ramas vivas o muertas de mangle rojo del Parque Ecológico Punta Sur. Hasta el momento no existen artículos sobre los ascomicetos en la isla; por lo que el presente estudio tiene como objetivo enlistar las especies de este grupo de hongos de tres Áreas Naturales Protegidas (ANPs) en la Reserva de la Biosfera Isla Cozumel, México.

\section{Materiales y Métodos}

\section{Área de estudio}

Se realizaron recolecciones en tres Áreas Naturales Protegidas de la Reserva de la Biosfera Isla Cozumel (Fig. 1):

1. Centro de Conservación y Educación Ambiental (CCEA), 20²8'59.19"N, 8657'21.80"O, vegetación de selva baja caducifolia. Las especies dominantes son Brassavola nodosa Lindl., Dioscorea floribunda M. Martens \& Galeotti, Diospyros nicaraguensis Standl., Disocactus flagelliformis (L.) Lem., Enriquebeltrania crenatifolia (Miranda) Rzed., Microgramma nitida (J. Sm.) A.R. Sm., Pithecellobium dulce (Roxb.) Benth., P. mangense J.F. Macbr. y Selenicereus testudo (Karw. ex Zucc.) Buxb. 

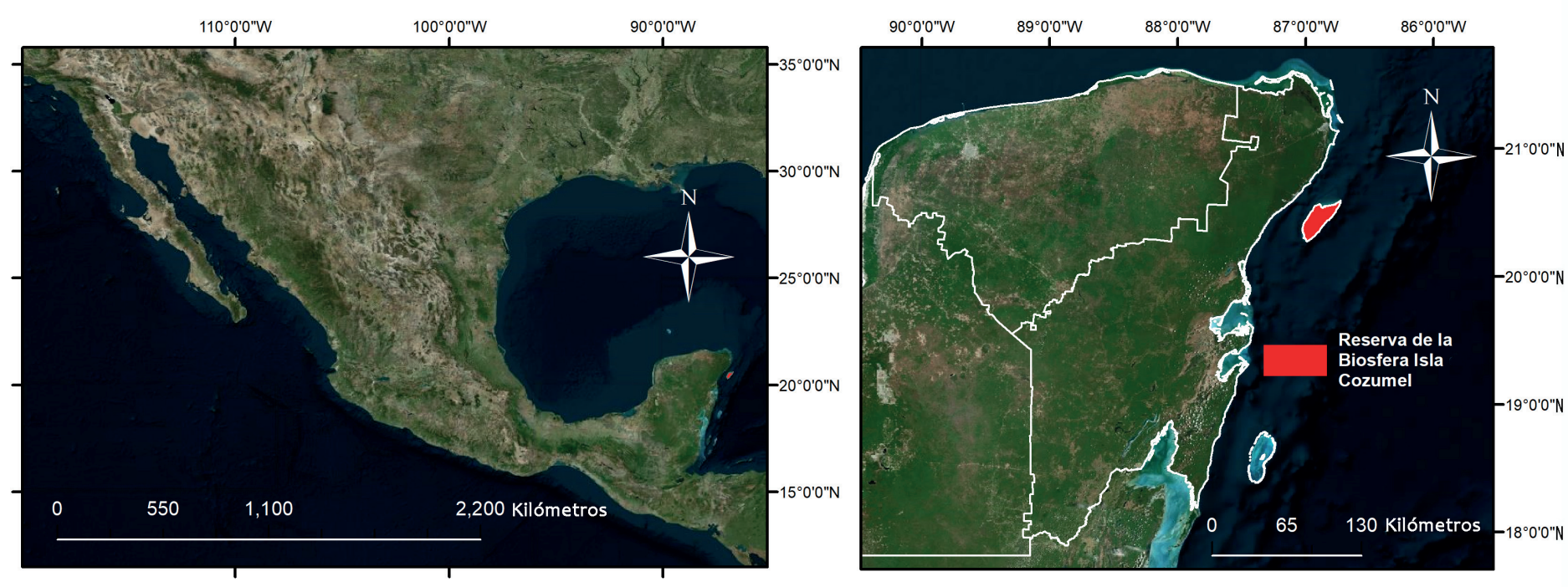

$87^{\circ} 0^{\prime} 0 " \mathrm{~W}$

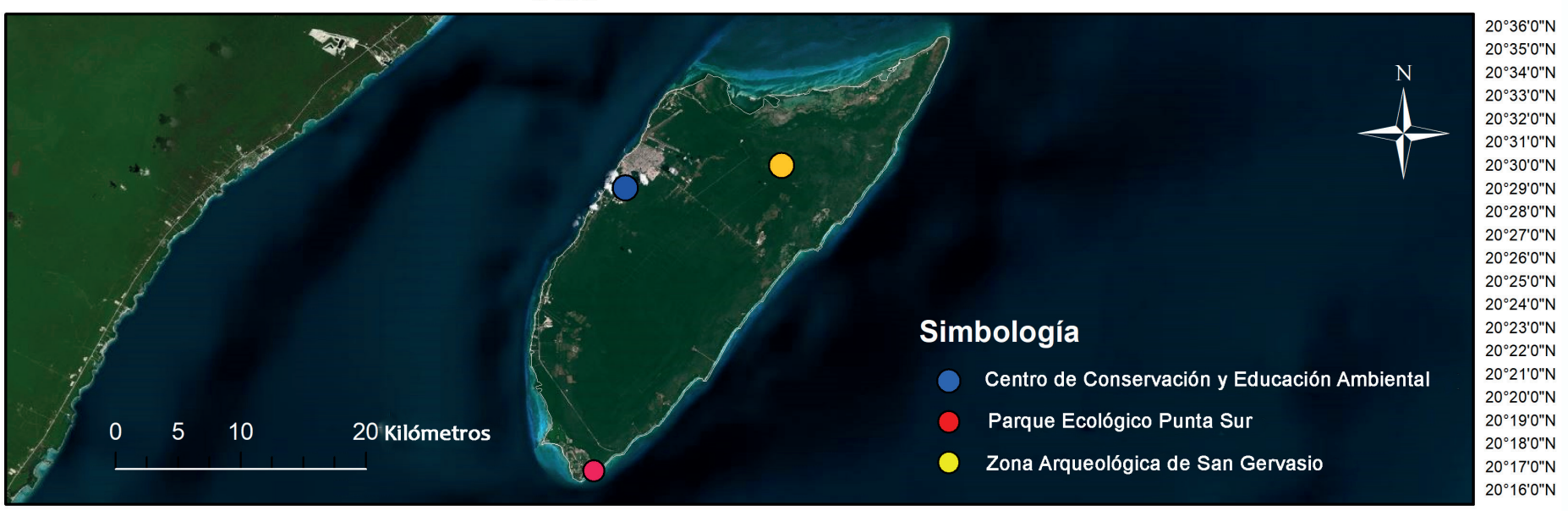

Figura 1: Ubicación de las localidades en la Reserva de la Biosfera Isla Cozumel, Quintana Roo, México.

2. Parque Ecológico Punta Sur (PEPS), $20^{\circ} 16^{\prime} 48^{\prime \prime} \mathrm{N}$, $86^{\circ} 58^{\prime} 44.39^{\prime \prime} \mathrm{O}$, vegetación de duna costera y manglar. Las especies presentes en las dunas costeras son Ambrosia hispida Pursh, Opuntia stricta (Haw.) Haw., Canavalia rosea (Sw.) DC., Sophora tomentosa L., Suriana maritima L., Hymenocallis caribaea (L.) Herb., Coccoloba uvifera, Thrinax radiata Lodd. ex Desf., Erithalis fruticosa L., Ernodea littoralis Sw., Salicornia bigelovii Torr., Enriquebeltrania crenatifolia y en el manglar se encuentran Rhizophora mangle, Avicennia germinans (L.) L., Laguncularia racemosa C.F. Gaertn. y Conocarpus erectus L.

3. Zona Arqueológica de San Gervasio, Chen-tuk (ZASG), $20^{\circ} 29^{\prime} 58.02 " \mathrm{~N}, 86^{\circ} 50^{\prime} 37.88^{\prime \prime} \mathrm{O}$, vegetación de sel- va mediana subcaducifolia. Las especies dominantes son Manilkara zapota (L.) P. Royen, Bursera simaruba, Calliandra belizensis (Britton \& Rose) Standl., Cedrela odorata L., Metopium brownei (Jacq.) Urb., Vitex gaumeri Greenm., Caesalpinia gaumeri Greenm., Ceiba aesculifolia (Kunth) Britten \& Baker f., Lysiloma latisiliquum (L.) Benth., Sideroxylon gaumeri (Pittier), Gliricidia sepium (Jacq.) Kunth ex Walp. y Coccoloba acapulcensis Standl. (Romero-Nájera, 2004).

\section{Trabajo de campo}

De 2018 a 2019 se realizaron cuatro exploraciones en las tres Áreas Naturales Protegidas de la Reserva de la Biosfera 
Isla Cozumel, mencionadas anteriormente, en enero y junio 2018, y en junio y octubre 2019.

El muestreo fue libre y sin restricciones según Angulo et al. (2006) y se presenta la distribución en México de las especies, y la descripción de la especie nueva de acuerdo con Reyes et al. (2020). Además, se tomaron fotografías in situ, utilizando cámaras Nikon D5500 (Nikon, Tokio, Japón) y Nikon D7000 (Nikon, Tokio, Japón).

\section{Trabajo de laboratorio}

Los especímenes recolectados se caracterizaron macro y micromorfológicamente de acuerdo con Chaverri et al. (2011a). Se consideraron el tamaño y forma del peritecio, ascas, ascosporas y células estériles. Se hicieron cortes longitudinales en preparaciones temporales utilizando alcohol al 70\% e hidróxido de potasio $(\mathrm{KOH})$ al 5\% para la extracción de pigmentos estromáticos de gran valor taxonómico. El estudio morfométrico se realizó en un microscopio óptico (MO) (K-7 Zeiss, Jena, Alemania) y para el detalle de la ornamentación de las esporas se utilizó microscopía electrónica de barrido (MEB) (JSM-5800LV, Jeol, Peabody, MA, EUA). Las especies se determinaron con las claves especializadas de Denison (1963), San Martín y Rogers (1989), Ju y Rogers (1996), San Martín et al. (1999), Carmarán et al. (2006), Hladki y Romero (2009), Chaverri et al. (2011a, b), PompaGonzález et al. (2011), Angelini y Medardi (2012), Fournier et al. (2015), Thambugala et al. (2016), y Barbosa-Reséndiz et al. (2020).

La terminología utilizada hace referencia al diccionario de Ulloa y Hanlin (2006). Los especímenes se depositaron en la Colección de Hongos “Dr. Gastón Guzmán Huerta” del Herbario de la Escuela Nacional de Ciencias Biológicas del Instituto Politécnico Nacional (ENCB).

\section{Resultados}

A continuación, se enlistan 40 especies de ascomicetos ubicadas en 25 géneros, 15 familias, nueve órdenes y cuatro clases, provenientes de las tres localidades que son Áreas Naturales Protegidas en la Reserva de la Biosfera Isla Cozumel. De estas, 15 especies se citan por primera vez para Quintana Roo, ocho para la isla. Bertia tropicalis Huhndorf (Bertiaceae) se cita por primera vez para México y Platystomum mexicanum Raymundo, Martínez-Pineda, A. Tun \& R.
Valenz. (Lophiostomataceae) se describe como nueva para la ciencia.

\section{Taxonomía}

\section{Ascomycota}

Dothideomycetes

Botryosphaeriales

\section{Botryosphaeriaceae}

Botryosphaeria dothidea (Moug.) Ces \& De Not. Comm. Soc. crittog. Ital. 1(4): 212. 1863.

TIPOS: FRANCIA. On Rosa sp., 1823, S. Mougeot s.n. (neotipo: S). SUIZA. Ticino, Crocifisso, Prunus sp., X.2000, B. Slippers s.n. (epitipo: PREM 57372).

= Xyloma rosae Schleich. ex DC., Fl. franç., Edn 3 (Paris) 5-6: 161. 1815.

इ Sphaeria dothidea Moug., in Fries, Syst. mycol. (Lundae) 2(2): 423.1823.

= Dothidea rosae (Schleich. ex DC.) Fr., Summa veg. Scand., Sectio Post. (Stockholm): 386. 1849.

= Botryosphaeria dothidea var. pluriguttata Brunaud, Bull. Soc. Sci. Nat. Ouest 3: 217. 1893.

= Dothiorella mali var. fructans Dearn., Mycologia 33(4): 361. 1941.

इCaumadothis dothidea (Moug.) Petr., Sydowia 24(1-6): 277. 1971.

Material examinado: MÉXICO. Quintana Roo, municipio Cozumel, Reserva de la Biosfera Isla Cozumel, Parque Ecológico Punta Sur, 16.VI.2018, T. Raymundo 8304 (ENCB), 8305 (ENCB).

Distribución en México: se ha reportado en Puebla (Valencia-Botín et al., 2003), Nayarit, Tabasco y Veracruz (Cibrián-Tovar, 2013). Se cita por primera vez para Quintana Roo. 
Lasiodiplodia theobromae (Pat.) Griffon \& Maubl. Bull. Soc. mycol. Fr. 25: 57. 1909.

TIPO: PAPUA NUEVA GUINEA. Madang, Jais Aben, de un fruto no identificado en las costas del arrecife de coral, $A$. Aptroot 1995 (neotipo: CBS H-21411), MBT176098, culture (ex-neotipo: CBS 164.96).

= Sphaeria glandicola Schwein., Trans. Am. phil. Soc., New Series 4(2): 214. 1832.

= Diplodia gossypina Cooke, Grevillea 7(43): 95. 1879.

= Physalospora rhodina Berk. \& M.A. Curtis, Grevillea 17(84): 92. 1889.

इ Botryodiplodia theobromae Pat., in Patouillard \& Lagerheim, Bull. Soc. mycol. Fr. 8(3): 136. 1892. TIPO: ECUADOR. En Theobroma cacao, Lagerheim s.n. (holotipo no encontrado y presumiblemente perdido).

= Phoma glandicola (Schwein.) Cooke, Grevillea 20(95): 85 . 1892.

= Cryptostictis glandicola (Schwein.) Starbäck, Bih. K. Svenska Vetensk. Akad. Handl., Afd. 3 19(2): 86. 1894.

= Macrophoma vestita Prill. \& Delacr., Bull. Soc. mycol. Fr. 10: 165. 1894.

= Diplodia cacaoicola Henn., Bot. Jb. 22: 80. 1895.

= Lasiodiplodia tubericola Ellis \& Everh., in Clendenin, Bot. Gaz. 21: 92. 1896.

= Botryodiplodia gossypii Ellis \& Barthol., J. Mycol. 8(4): 175. 1902.

= Botryodiplodia elasticae Petch, Ann. R. bot. Gdns Peradeniya 3(1): 7. 1906.

= Chaetodiplodia grisea Petch, Ann. R. bot. Gdns Peradeniya 3(1): 6. 1906.

= Lasiodiplodia nigra Griffon \& Maubl., Bull. Soc. mycol. Fr. 25: 57. 1909.

= Diplodia natalensis Pole-Evans, Transvaal Dept. of Agricult. Sci. Bull. 4: 15. 1911.

$=$ Thyridaria tarda C.K. Bancr., Bull. Dept. Agric. Fed. Malay Stat. 9: (1). 1911.

= Diplodia tubericola (Ellis \& Everh.) Taubenh., Am. J. Bot. 2(7): 328. 1915.

= Lasiodiplodia triflorae B.B. Higgins, Bull. Georgia Exp. Stn 118: 16. 1916.

= Pyreniella rhodina (Berk. \& M.A. Curtis) Theiss., Verh. zool.- bot. Ges. Wien 66: 392. 1916.

= Diplodia ananassae Sacc., Atti Accad. Sci. Ven.-Trent.-Istr. 10: 75.1917.

E Diplodia theobromae (Pat.) W. Nowell, Diseases of Crop Plants in the Lesser Antilles: 158. 1923.

= Botryodiplodia tubericola (Ellis \& Everh.) Petr., Annls mycol. 21(3-4): 332. 1923.

= Physalospora gossypina F. Stevens, Mycologia 17(5): 200. 1925.

= Botryodiplodia ananassae (Sacc.) Petr., Annls mycol. 27(56): 365. 1929.

= Physalospora glandicola (Schwein.) N.E. Stevens, Mycologia 25(6): 504. 1933.

= Lasiodiplodiella triflorae (B.B. Higgins) Zambett., Bull. trimest. Soc. mycol. Fr. 70(3): 229. 1955.

= Botryosphaeria rhodina (Berk. \& M.A. Curtis) Arx, Gen. Fungi Sporul. Cult. (Lehr): 143. 1970.

Material examinado: MÉXICO. Quintana Roo, municipio Cozumel, Reserva de la Biosfera Isla Cozumel, Parque Ecológico Punta Sur, 16.VI.2018, T. Raymundo 7672 (ENCB); loc. cit., 14.X.2019, T. Raymundo 8306 (ENCB).

Distribución en México: Campeche, Ciudad de México, Colima, Estado de México, Guerrero, Guanajuato, Jalisco, Michoacán, Nayarit, Puebla, Querétaro, Tabasco, Tlaxcala (Cibrián-Tovar et al., 2007), Veracruz (Medel y Chacón, 1988) y Sonora (Raymundo et al., 2017a). Se cita por primera vez para Quintana Roo.

Hysteriales

Hysteriaceae

Gloniella tropicalis I. Álvarez, Raymundo \& R. Valenz., Act. Bot. Mex. 116 (4): 54.2016.

TIPO: MÉXICO. Oaxaca, Parque Nacional Lagunas de Chacahua, km 4 del camino de terracería, Los Azufres - La Grúa, 12.X.2011, T. Raymundo 4093 (ENCB!).

Material examinado: MÉXICO. Quintana Roo, municipio Cozumel, Reserva de la Biosfera Isla Cozumel, Zona 
Arqueológica de San Gervasio, Chen-tuk, 17.VI.2018, T. Raymundo 7685 (ENCB).

Distribución en México: Oaxaca (Álvarez et al., 2016). Se cita por primera vez para Quintana Roo.

Rhytidhysteron neorufulum Thambug. \& K.D. Hyde, Cryptogamie Mycologie 37(1): 110. 2016. Fig. 2A.

TIPO: TAILANDIA. Provincia de Chiang Rai, Centro de Investigación Hortícola Chiangrai, 2012, K. M. Thambugala 3 (holotipo: MFLU, isotipo: PDD).

Material examinado: MÉXICO. Quintana Roo, municipio Cozumel, Reserva de la Biosfera Isla Cozumel, Centro de Conservación y Educación Ambiental, 19.I.2018, A. Cobos-ViIlagrán 915 (ENCB); Parque Ecológico Punta Sur, 16.VI.2018, A. Cobos-Villagrán 1816 (ENCB); Zona Arqueológica de San Gervasio, Chen-tuk, 17.VI.2018, A. Bernal 132 (ENCB); loc. cit., A. Cobos-Villagrán 1840 (ENCB), 1841 (ENCB), 1843 (ENCB); loc. cit., 21.I.2018, T. Raymundo 7337 (ENCB); loc. cit., 17.VI.2018, T. Raymundo 7688 (ENCB).

Distribución en México: Campeche, Colima, Jalisco, Nayarit, Oaxaca, Quintana Roo y San Luis Potosí (Cobos-Villagrán et al., 2020).

Rhytidhysteron rufulum (Spreng.) Speg. An. Soc. Cient. Arg. 90(1-6): 177. 1921. Fig. 2B.

इ Hysterium rufulum Spreng., Kongl. Svenska Vetenskaps Academiens Handlingar 41: 50. 1820. TIPO: PUERTO RICO. C. Sprengel s.n. (especímen tipo no designado ni se menciona en qué herbario se depositó).

$\equiv$ Tryblidiella rufula (Spreng.) Sacc. Sacc., Syll. Fung. (Abellini) 2: 757.1883.

इ Triblidium rufulum (Spreng.) Ellis \& Everh., N. Amer. Pyren. (Newfield): 690. 1892.

三 Brunaudia rufula (Spreng.) Kuntze (as 'Bruneaudia') Revis. Gen. Pl. (Leipzig) 3(3): 447. 1898.

Material examinado: MÉXICO. Quintana Roo, municipio Cozumel, Reserva de la Biosfera Isla Cozumel, Centro de
Conservación y Educación Ambiental, 19.I.2018, A. CobosVillagrán 915 (ENCB); Parque Ecológico Punta Sur, 16.VI.2018, A. Cobos-Villagrán 1811 (ENCB), 1817 (ENCB), 1820 (ENCB), 1821 (ENCB), 1823 (ENCB), 1824 (ENCB), 1825 (ENCB); loc. cit., T. Raymundo 7660 (ENCB), 7663 (ENCB); Zona Arqueológica San Gervasio, Chen-tuk, 17.VI.2018, A. Cobos-Villagrán 1844 (ENCB), 1845 (ENCB), 1861 (ENCB); loc. cit., T. Raymundo 7690 (ENCB).

Distribución en México: se ha citado de los estados de Sonora (Méndez-Mayboca et al., 2010), Chiapas, Guerrero, Nuevo León, Puebla, Querétaro, Quintana Roo, Sonora, Tabasco, Tamaulipas, Veracruz, Yucatán (Chacón et al., 2014), Oaxaca (Raymundo et al., 2014; Álvarez et al., 2016), Campeche, Colima, Estado de México, Hidalgo, Jalisco, Nayarit y San Luis Potosí (Cobos-Villagrán et al., 2020).

Patellariales

Patellariaceae

Patellaria atrata (Hedw.) Fr., Syst. mycol. (Lundae) 2(1): 158. 1822. Fig. 2 C.

TIPO: ALEMANIA. En ramas de Tilia Hedwig. (holotipo: no se indica en qué herbario está depositado el tipo).

इ Lichen atratus Hedw., Descr. Micr.-anal. Musc. Frond. (Lipsiae) 2:61. 1789.

= Peziza patellaria Pers., Syn. meth. fung. (Göttingen) 2: 670 . 1801.

इ Peziza atrata (Hedw.) Schumach., Enum. pl. (Kjøbenhavn) 2: 417. 1803.

= Peziza patellaria var. foveolans Pers., Mycol. eur. (Erlanga) 1: 307.1822.

= Peziza patellaria var. picea Pers., Mycol. eur. (Erlanga) 1: 307.1822.

= Peziza patellaria var. valvata Pers., Mycol. eur. (Erlanga) 1: 307. 1822.

इ Lecanidion atratum (Hedw.) Endl., Flora Pason 1: 46. 1830.

三 Cycledium atratum (Hedw.) Wallr. (as "Cycledum atrum"), Fl. crypt. Germ. (Norimbergae) 2: 511. 1833.

= Opegrapha atra f. denigrata (Ach.) Moug., Bull. Soc. bot. Fr. 

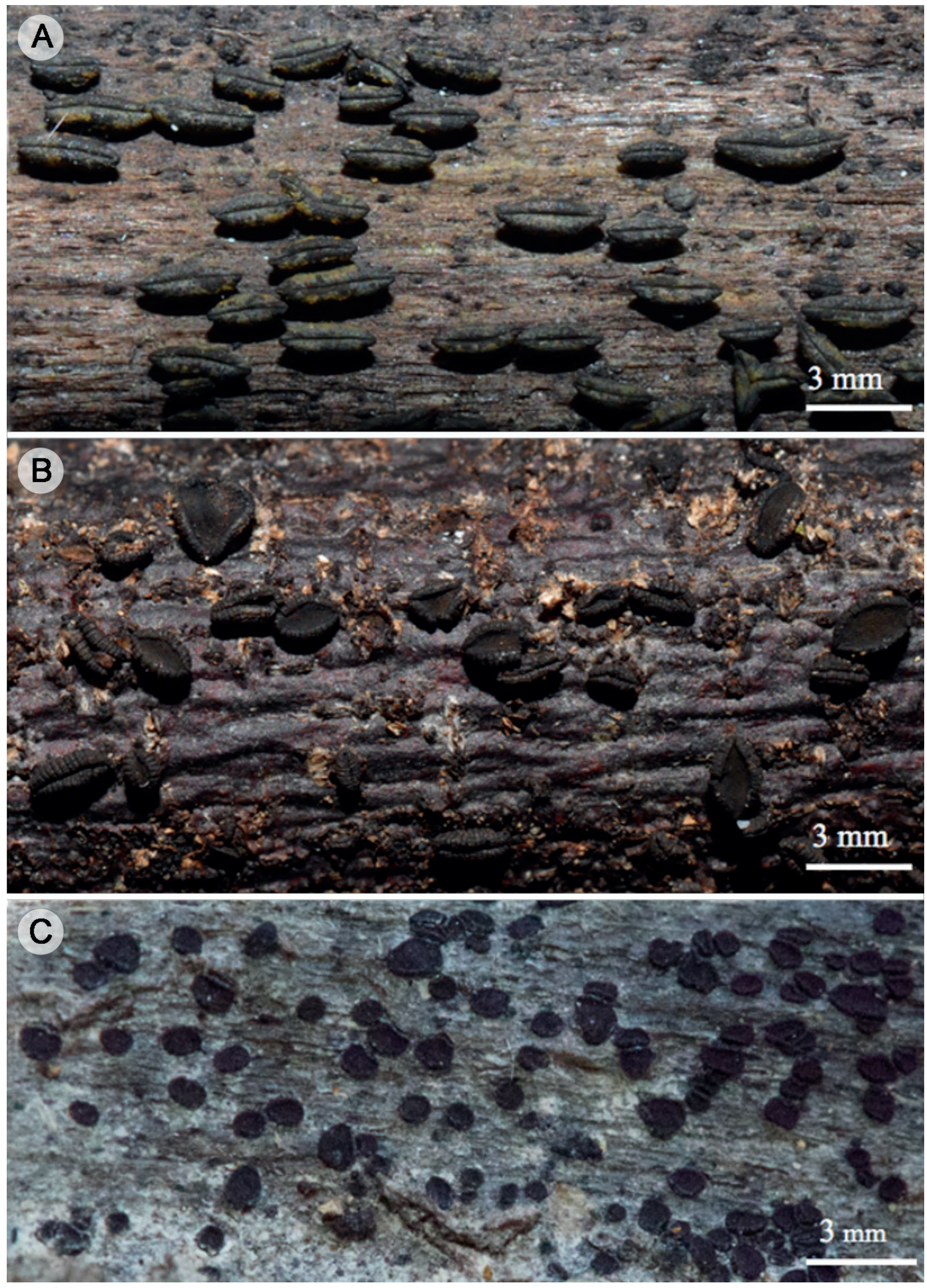

Figura 2: Dothideomycetes: A. Rhytidhysteron neorufulum Thambug. \& K.D. Hyde; B. R. rufulum (Spreng.) Speg.; C. Patellaria atrata (Hedw.) Fr. 
4(4): 259. 1857.

= Ucographa lecanactis A. Massal. ex Arnold, Flora, Regens-

burg 45(25): 394. 1862.

= Patellaria indigotica Cooke \& Peck, in Peck, Ann. Rep. Reg.

Univ. St. N.Y. 25: 98. 1873.

= Lecanidion indigoticum (Cooke \& Peck) Sacc., Syll. fung. (Abellini) 8: 797. 1889.

इUcographa atrata (Hedw.) Arnold, Ber. bayer. bot. Ges. 1: 133. 1891.

= Patellaria maura Massee Bull. Misc. Inf., Kew: 131. 1898.

三 Pragmopora atrata (Hedw.) Körb. ex Mussat, in Saccardo, Syll. fung. (Abellini) 15: 287. 1901.

= Patellaria atrata f. indigotica (Cooke \& Peck) Rehm, Annls mycol. 4(4): 337. 1906.

= Patellaria atrata f. hedericola Bubák, Annls mycol. 12(2):

206. 1914.

= Patellaria masseei Rodway (as "masseea"), Pap. Proc. R. Soc. Tasm.: 156. 1921.

= Bilimbia sublubens Paulson, Trans. Br. mycol. Soc. 12(2-3): 88. 1927.

= Bacidia sublubens (Paulson) Zahlbr., Cat. Lich. Univers. 8: 409. 1932.

Material examinado: MÉXICO. Quintana Roo, municipio Cozumel, Reserva de la Biosfera Isla Cozumel, Parque Ecológico Punta Sur, 15.X.2019, T. Raymundo 8326 (ENCB).

Distribución en México: Sonora (Méndez-Mayboca et al., 2010), Chiapas, Veracruz (Chacón y Tapia, 2016) y Quintana Roo (García-Martínez et al., 2021b).

Pleosporales

Anteaglonaceae

Anteaglonium abbreviatum (Schwein.) Mugambi \& Huhndorf, Systematics and Biodiversity 7(4): 460. 2009.

TIPO: ESTADOS UNIDOS DE AMÉRICA. Pennsylvania, Northampton County, Bethlehem, L. D. Schweinitz 2094 (holotipo: $\mathrm{PH}$ ).
इ Hysterium abbreviatum Schwein., Trans. Am. phil. Soc., New Series 4(2): 244. 1832.

इ Lophiostoma abbreviatum (Schwein.) Sacc., Syll. fung. (Abellini) 2: 710. 1883.

三 Glonium abbreviatum (Schwein.) M.L. Lohman, Bull. Torrey bot. Club 64: 64. 1937.

Material examinado: MÉXICO. Quintana Roo, municipio Cozumel, Reserva de la Biosfera Isla Cozumel, Zona Arqueológica de San Gervasio, Chen-tuk, 17.VI.2018, T. Raymundo 7684 (ENCB).

Distribución en México: Oaxaca (Álvarez et al., 2016). Se cita por primera vez para Quintana Roo.

\section{Lophiostomataceae}

Platystomum mexicanum Raymundo, Martínez-Pineda, A. Tun \& R. Valenz., sp. nov. Figs. 3A-D.

TIPO: MÉXICO. Quintana Roo, municipio Cozumel, Parque Ecológico Punta Sur, $28^{\circ} 16^{\prime} 48^{\prime \prime} \mathrm{N}, 86^{\circ} 58^{\prime} 44.39^{\prime \prime} \mathrm{O}$, 16.VI.2018, T. Raymundo 7671 (holotipo: ENCB!). Mycobank: MB839198.

Differunt per P. compresum (Pers.) Trevis. Ascomata $600-700 \times 400-600 \mathrm{~mm}$, ascis bitunicatis, $116-120 \times 10-12$ $\mu \mathrm{m}$ longis, octosporis, ascosporis 16-18 $\times 6-8 \mu \mathrm{m}$, ellipticis, octonae, 1-3 septatis transversis, 2-4 septatis verticalis orientibus.

Ascoma 600-700 $\times$ 400-600 $\mu \mathrm{m}$, subgloboso, inmerso, papila errumpente a través de la superficie del hospedero, negro, carbonoso con ostiolo central papilado reducido a un poro rodeado por una pared pseudoparenquimatosa, 20-30 $\mu \mathrm{m}$ de grosor, marrón obscuro, formado por células isoadiamétricas a angulares, 5-8 $\mu \mathrm{m}$ de diámetro; pseudoparáfisis 1-1.5 $\mu \mathrm{m}$ de diámetro, ramificadas, embebidas en una matriz gelatinosa; ascas 116-120 × 10-12 $\mu \mathrm{m}$, cilíndrico-clavadas, bitunicadas, octosporadas, uniseriadas, hialinas, con ápice redondeado y estípite corto; ascosporas 16$18 \times 6-8 \mu \mathrm{m}$, elipsoides, hialinas cuando inmaduras, ámbar 

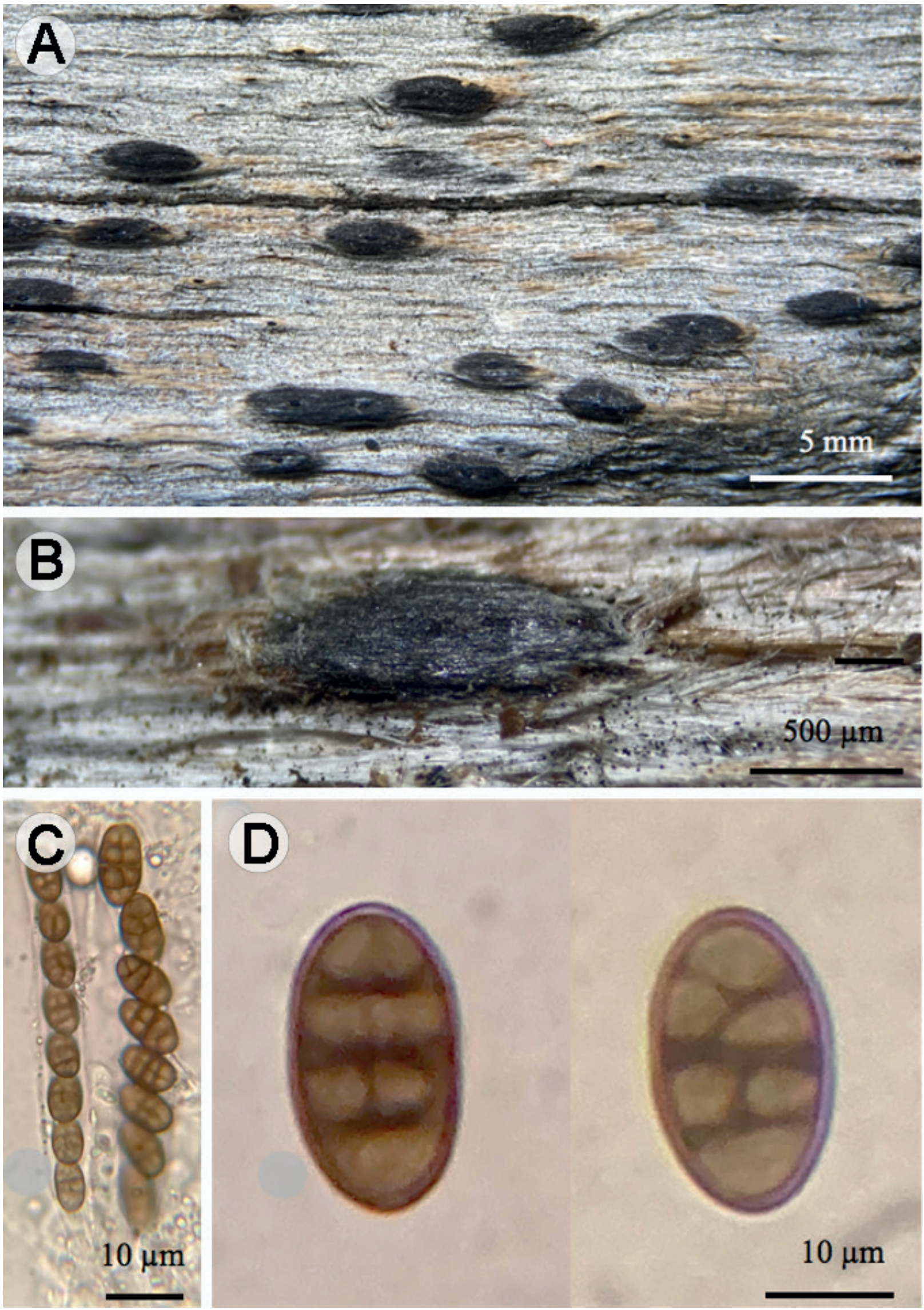

Figura 3: Platystomum mexicanum Raymundo, Martínez-Pineda, A. Tun \& R. Valenz.: A-B. ascomas; C. ascas; D. ascosporas. 
hasta marrón obscuro al madurar, muriformes, 1-3 septos transversales, 2-4 septos verticales, paredes gruesas y lisas, septo central sin constreñir.

Hábitat: crece gregario sobre ramas de Manilkara zapota (Sapotaceae).

Etimología: hace referencia al país de donde se describe.

Distribución: solo se conoce de la localidad tipo.

Notas taxonómicas: se distingue por las esporas muriformes de 16-18 × 6-8 $\mu \mathrm{m}$, elipsoides, con el septo central sin constreñir y sin una vaina gelatinosa como ocurre en otras especies del género. Otras especies similares son $P$. compressum (Pers.) Trevis y P. actinidiae Thambugala, Jayasiri, Kaz. Tanaka \& K.D. Hyde, aunque se diferencian porque la primera presenta esporas de 17-32.5 × 7-9.5 $\mu \mathrm{m}$ con 5-7 septos tranversales y 1-3 verticales, crece sobre Salix sp. en Hungría e Italia, mientras que la segunda produce esporas de 14-16 $\times 6-8 \mu \mathrm{m}$ con tres septos transversales y uno vertical, y crece sobre Actinidia deliciosa C.F. Liang \& A.R. Ferguson en Japón.

\section{Macrovalsariaceae}

Macrovalsaria megalospora (Mont.) Sivan., Trans. Br. Mycol. Soc. 65(3): 400. 1975.

TIPO: GUAYANA FRANCESA. Cayenne, in corticibus emortiorum circa Cayennae lecta, Leprieur 239 (holotipo: K).

इSphaeria megalospora Mont., Annales des Sciences Naturelles Botanique 14: 324. 1849.

= Amphisphaeria megalospora (Mont.) Sacc., Syll. Fung. (Abellini) 1: 724. 1882.

三 Melogramma megalospora (Mont.) Cooke, Grevillea 13(68): 109. 1885.

Material examinado: MÉXICO. Quintana Roo, municipio Cozumel, Reserva de la Biosfera Isla Cozumel, Centro de Conservación y Educación Ambiental, 20.I.2018, T. Raymundo 7281 (ENCB).
Distribución en México: Veracruz (Chacón et al., 2014). Se cita por primera vez para Quintana Roo.

\section{Leotiomycetes}

Cyttariales

\section{Cordieritidaceae}

Unguiculariopsis ravenelii (Berk. \& M.A. Curtis) W.Y. Zhuang \& Korf, Mycotaxon 29: 395. 1987. Fig. 4A.

TIPO: ESTADOS UNIDOS DE AMÉRICA. Carolina del Sur, sobre Rhytidhysteron rufulum (como Patellaria nigrocinnabarina Schwein.), Ravenel 551 (neotipo: CUP-061819).

इ Peziza ravenelii Berk. \& M.A. Curtis, Grevillea 3(28): 152. 1875.

三 Cenangium ravenelii (Berk. \& M.A. Curtis) Sacc., Syll. Fung. (Abellini) 8: 568. 1889.

三 Encoeliella ravenelii (Berk. \& M.A. Curtis) Höhn., Sber. Akad. Wiss. Wien, Math.-naturw. Klasse., Abt. 1 119: 619. 1910.

= Pithyella hamata Chenant., Bull. Soc. Mycol. Fr. 34: 39. 1918.

= Unguiculariopsis ravenelii subsp. hamata (Chenant.) W.Y. Zhuang, Mycotaxon 32(1): 53. 1988.

Material examinado: MÉXICO. Quintana Roo, municipio Cozumel, Reserva de la Biosfera Isla Cozumel, Zona Arqueológica de San Gervasio, Chen-tuk, sobre Rhytidhysteron rufulum, 21.I.2018, T. Raymundo 7334 (ENCB).

Distribución en México: Colima, Jalisco, Oaxaca, Puebla, Tabasco y Tamaulipas (Raymundo et al., 2020). Se cita por primera vez para Quintana Roo.

Pezizomycetes

Pezizales

Sarcoscyphaceae 

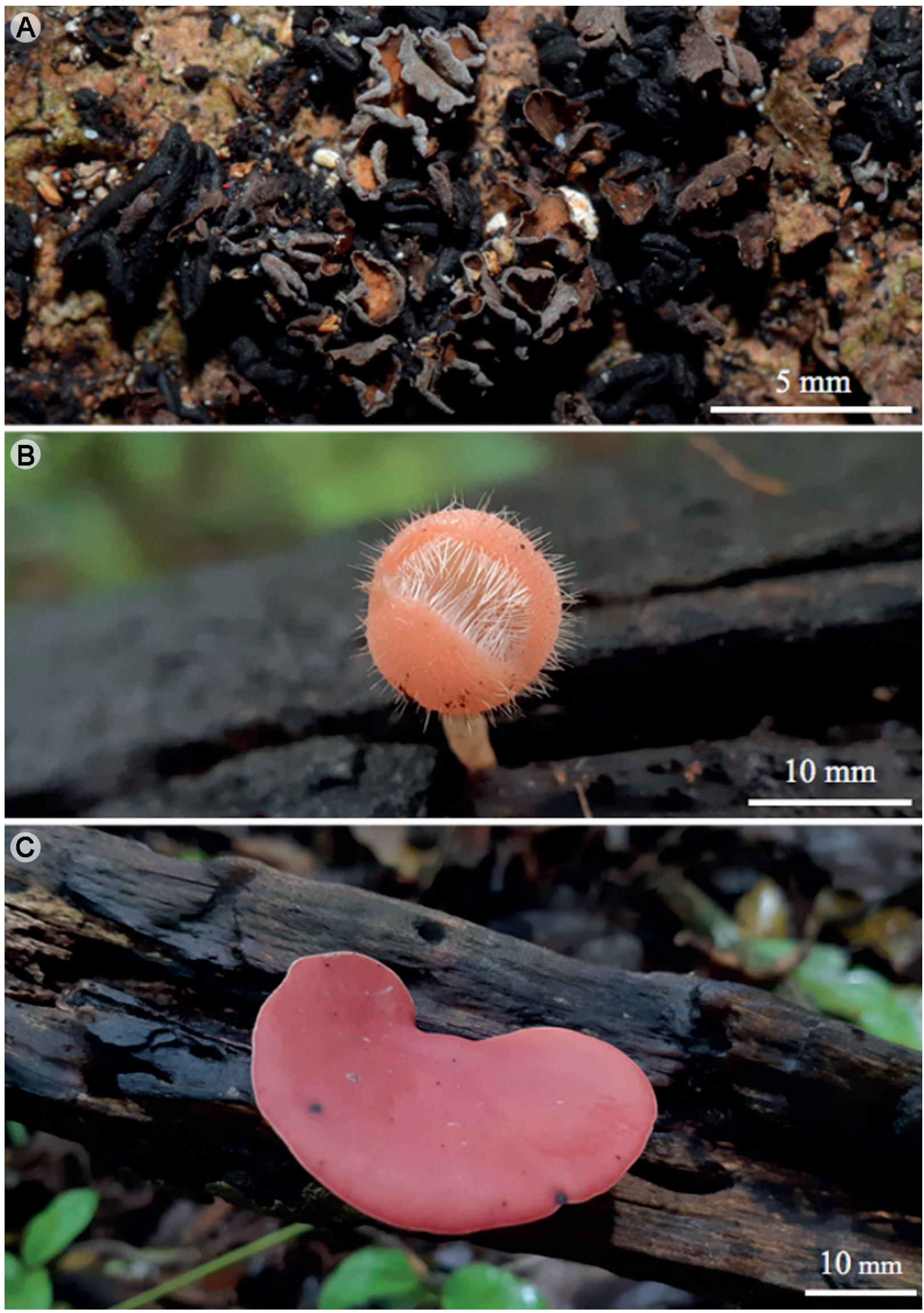

Figura 4: Leotiomyctes y Pezizomycetes: A. Unguiculariopsis ravenelii (Berk. \& M.A. Curtis) W.Y. Zhuang \& Korf; B. Cookeina tricholoma (Mont.) Kuntze; C. Phillipsia domingensis (Berk.) Berk. ex Denison. 
Cookeina tricholoma (Mont.) Kuntze 1891 Revis. gen. pl. (Leipzig) 2: 849. 1891. Fig. 4B.

TIPO: BRASIL. Río de Janeiro, M. Gaudichaud 44 (lectotipo: PC).

इ Peziza tricholoma Mont., Annls Sci. Nat., Bot., sér. 2(2):

77. 1834.

= Peziza tricholoma var. minor Mont., Annls Sci. Nat., Bot., sér. 2(2): 77. 1834.

इ Lachnea tricholoma (Mont.) Pat., in Patouillard \& Gaillard, Bull. Soc. mycol. Fr. 4(2): 98. 1888.

इ Trichoscypha tricholoma (Mont.) Cooke, in Saccardo, Syll. fung. (Abellini) 8: 160. 1889.

इ Pilocratera tricholoma (Mont.) Henn., Bot. Jb. 17: 9. 1893.

= Pilocratera tricholoma var. celebica Henn., in Warburg, Monsunia 1: 23. 1900.

Material examinado: MÉXICO. Quintana Roo, municipio Cozumel, Reserva de la Biosfera Isla Cozumel, Centro de Conservación y Educación Ambiental, 20.I.2018, A. A. Tun 1 (ENCB); Zona Arqueológica de San Gervasio, Chen-tuk, 21.I.2018, A. A. Tun 8 (ENCB).

Distribución en México: Chiapas (Robles-Porras et al., 2006), Quintana Roo (Guzmán, 2003), Jalisco (SánchezJacome y Guzmán-Dávalos, 2011), Oaxaca, Veracruz (Welden y Guzmán, 1978; Raymundo et al., 2014), Campeche, Jalisco, Oaxaca y San Luis Potosí (Ortega-López et al., 2019). Se registra por primera vez para la Isla Cozumel.

Phillipsia domingensis (Berk.) Berk. ex Denison, Mycologia 61(2): 293. 1969. Fig. 4C.

TIPO: REPUBLICA DOMINICANA. On wood, Salle 42 (holotipo: K 122223)

ミPeziza domingensis Berk., Ann. Mag. nat. Hist., Ser. 2 9: 201. 1852

三 Otidea domingensis (Berk.) Sacc., Syll. fung. (Abellini) 8: 97. 1889.

三 Molliardiomyces domingensis Paden, Can. J. Bot. 62(3): 214. 1984
Material examinado: MÉXICO. Quintana Roo, municipio Cozumel, Reserva de la Biosfera Isla Cozumel, Centro de Conservación y Educación Ambiental, 16.VI.2018, A. A. Tun 2 (ENCB); Zona Arqueológica de San Gervasio, Chen-tuk, 17.VI.2018, A. A. Tun 9 (ENCB).

Distribución en México: Chiapas, Hidalgo, Jalisco, Oaxaca y San Luis Potosí (Ortega-López et al., 2019), Estado de México (Guzmán-Dávalos y Guzmán, 1979), Hidalgo (Raymundo et al., 2016), Jalisco (Guzmán y García-Saucedo, 1973; Guzmán-Dávalos et al., 1983), Morelos (Portugal et al., 1985), Oaxaca (Welden y Guzmán, 1978; Raymundo et al., 2014), Quintana Roo (Guzmán, 2003), Tamaulipas (Heredia, 1989) y Veracuz (Welden y Guzmán, 1978). Se registra por primera vez para la Isla Cozumel.

\section{Sordariomycetes}

\section{Coronophorales}

\section{Bertiaceae}

Bertia tropicalis Huhndorf, A.N. Mill. \& F.A. Fernández, Mycol. Res. 108(12): 1387. 2004. Figs. 5A-D.

TIPO: ESTADOS UNIDOS DE AMÉRICA. Puerto Rico, Caribbean National Forest, El Verde Research Area, 3.X.1995, sobre corteza de ramas superiores de Nectandra turbacensis, S. M. Huhndorf 1707 (holotipo: F).

Peritecios ostiolados, $800-1100 \times 700-850 \mu \mathrm{m}$, parte basal $450-550 \mu \mathrm{m}$ de ancho, solitarios a gregarios, forman grupos pequeños o grandes, sobre una base estéril, turbinados, colapsados en especímenes secos, marrón obscuro a negros, superficie tuberculada; peridio $100-120 \mu \mathrm{m}$ de grosor en los lados, parte apical 150-200 $\mu \mathrm{m}$ de grosor, parte basal 230-250 $\mu \mathrm{m}$ de grosor, textura globular, células angulares a pseudoparenquimáticas, 10-15 $\mu$ m de diámetro, marrón obscuro, poros de Munk presentes; paráfisis infladas, hialinas, septadas, no ramificadas, 13.6-16 $\mu \mathrm{m}$ de diámetro; ascas 200-250 × 14-15 $\mu \mathrm{m}$, cilíndricas a clavadas, base largamente estipitada, 100-135 $\mu \mathrm{m}$, octosporadas, biseriadas; ascosporas 22-37 × 5-10 $\mu \mathrm{m}$, cilíndricas, curvadas en la madurez, genicu- 

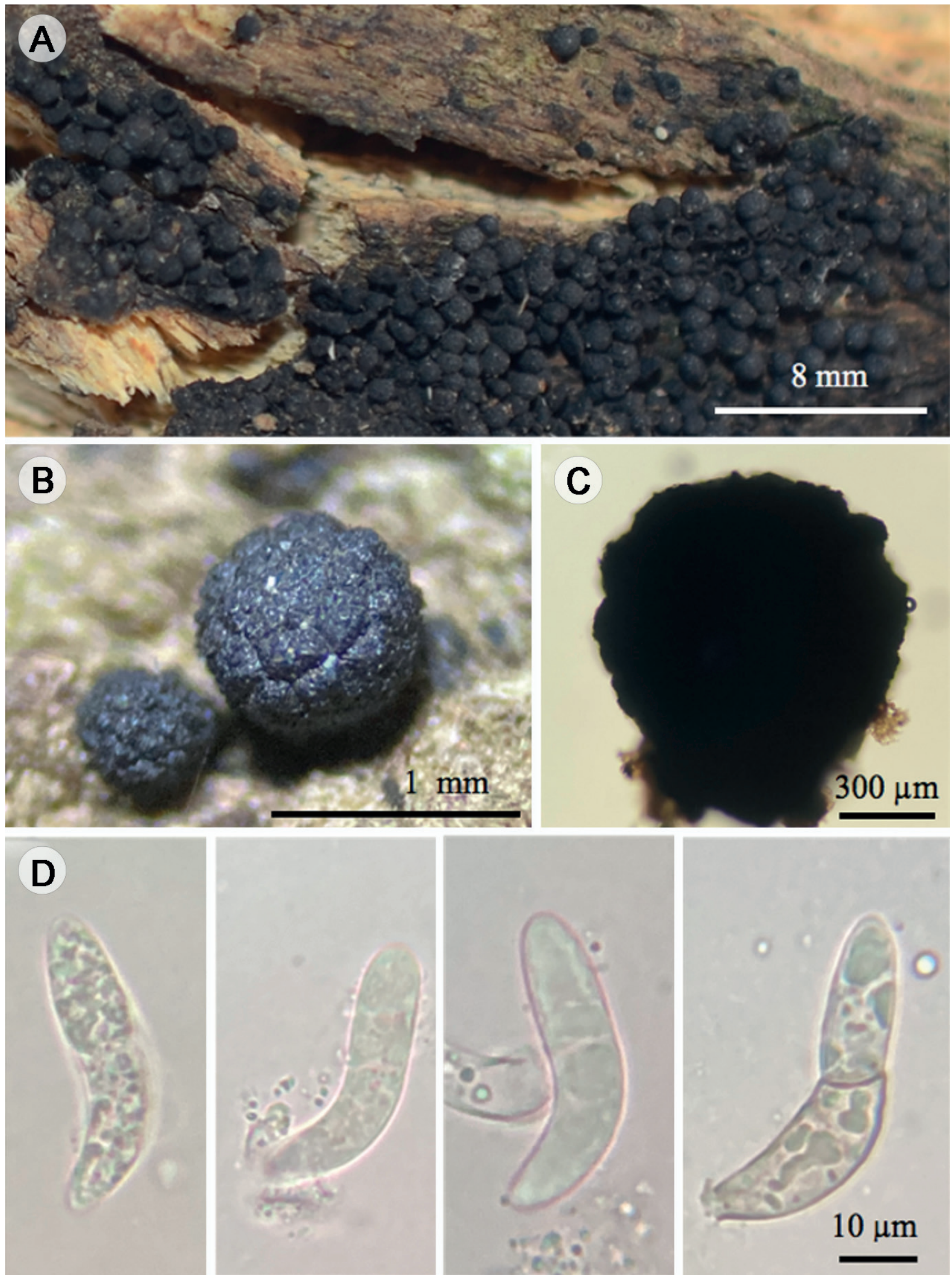

Figura 5: Bertia tropicalis Huhndorf, A.N. Mill. \& F.A. Fernández: A. ascomas gregarios; B. peritecios inmaduro y maduro; C. peritecios turbinados; D. ascosporas. 
ladas, hialinas, unicelulares cuando jóvenes, bicelulares cuando maduras, septo central, lisas, paredes delgadas.

Hábitat: crece sobre madera descortezada en descomposición en bosque tropical caducifolio.

Material examinado: MÉXICO. Quintana Roo, municipio Cozumel, Reserva de la Biosfera Isla Cozumel, Centro de Conservación y Educación Ambiental, 20.I.2018, T. Raymundo 7286 (ENCB); loc. cit., R. Valenzuela 17938 (ENCB).

Distribución: se ha descrito de Costa Rica, Estados Unidos de América (Puerto Rico), Guyana Francesa, Jamaica y Panamá (Huhndorf et al., 2004), Costa Rica (Chaverri et al., 2011a). Se cita por primera vez para México.

Notas taxonómicas: esta especie se caracteriza por el tamaño, forma y color de sus ascosporas y la presencia de paráfisis infladas, septadas y no ramificadas. Se separa de Bertia dydyma (Speg.) Mugambi \& Huhndorf y B. multiseptata (Sivan.) Huhndorf, A.N. Mill. \& F.A. Fernández por que sus ascosporas son de tres o más septos y de color marrón, a diferencia de $B$. tropicalis que presenta las ascosporas con un septo y hialinas. Otra especie afín por sus ascosporas hialinas y geniculadas es Bertia triseptata Mugambi \& Huhndorf, pero tiene también tres septos en éstas. Bertia gigantispora (Nannf.) Mugambi \& Huhndorf y B. moriformis (Tode) De Not. con ascosporas hialinas y un septo, se separan de $B$. tropicalis por las esporas rectas y de mayor tamaño. Finalmente, dos especies afines a $B$. tropicalis por la similitud en tamaño, forma y color de las ascosporas son B. hainanensis Lar.N. Vassiljeva, Hay X. Ma, Chernyshev \& S.L. Stephenson y B. ngongensis Mugambi \& Huhndorf, pero la primera se separa por las paráfisis filamentosas y ascosporas muy angostas, 4.8-5.5 $\mu \mathrm{m}$ de diámetro y 5-10 $\mu \mathrm{m}$ en $B$. tropicalis y la segunda por tener dos capas diferenciadas en el peridio del peritecio y paráfisis ligeramente infladas y ramificadas (Mugambi y Huhndorf, 2010; Vassiljeva et al., 2015).

Hypocreales

Bionectriaceae
Bionectria ochroleuca (Schwein.) Schroers \& Samuels, Z. Mykol. 63(2): 151. 1997. Fig. 6A.

TIPO: ESTADOS UNIDOS DE AMÉRICA. North Carolina, Salem, on bark, 1821, L. D. Schweinitz 1418 (holotipo: PH, Collins Set 169; lectotipo: NY).

इ Sphaeria ochroleuca Schwein., Trans. Am. phil. Soc., New Series 4(2): 204. 1834.

इ Nectria ochroleuca (Schwein.) Berk., Grevillea 4(29): 16. 1875.

= Nectria vulgaris Speg., Anal. Soc. cient. Argent. 12(5): 210. 1881.

= Verticillium tubercularioides Speg. (as 'tubercularioide'), Anal. Soc. cient. Argent. 12(5): 210. 1881.

इ Cucurbitaria ochroleuca (Schwein.) Kuntze, Revis. gen. pl. (Leipzig) 3(3): 461. 1898.

= Cucurbitaria vulgaris (Speg.) Kuntze, Revis. gen. pl. (Leipzig) 3(3): 460. 1898.

इCreonectria ochroleuca (Schwein.) Seaver, Mycologia 1(5): 190. 1909.

= Nectria ochroleuca var. longispora Wollenw., Angew. Bot. 8: 184.1926.

= Polystigma vulgare (Speg.) Gola, L'Erbario Micologico di P.A. Saccardo: 159. 1930.

= Nectria gliocladioides Smalley \& H.N. Hansen, Mycologia 49(4): 533. 1957.

Material examinado: MÉXICO. Quintana Roo, municipio Cozumel, Reserva de la Biosfera Isla Cozumel, Centro de Conservación y Educación Ambiental, 20.I.2018, T. Raymundo 7292 (ENCB); Zona Arqueológica de San Gervasio, Chentuk, 17.VI.2018, T. Raymundo 7669 (ENCB); Parque Ecológico Punta Sur, 14.X.2019, T. Raymundo 8307 (ENCB).

Distribución en México: Hidalgo (Raymundo et al., 2017b). Se cita por primera vez para Quintana Roo.

\section{Hypocreaceae}

Hypocreopsis macrostoma (Berk. \& M.A. Curtis) E. Müll. in Müller \& von Arx, Beitr. Kryptfl. Schweiz 11(2): 650. 1962. 

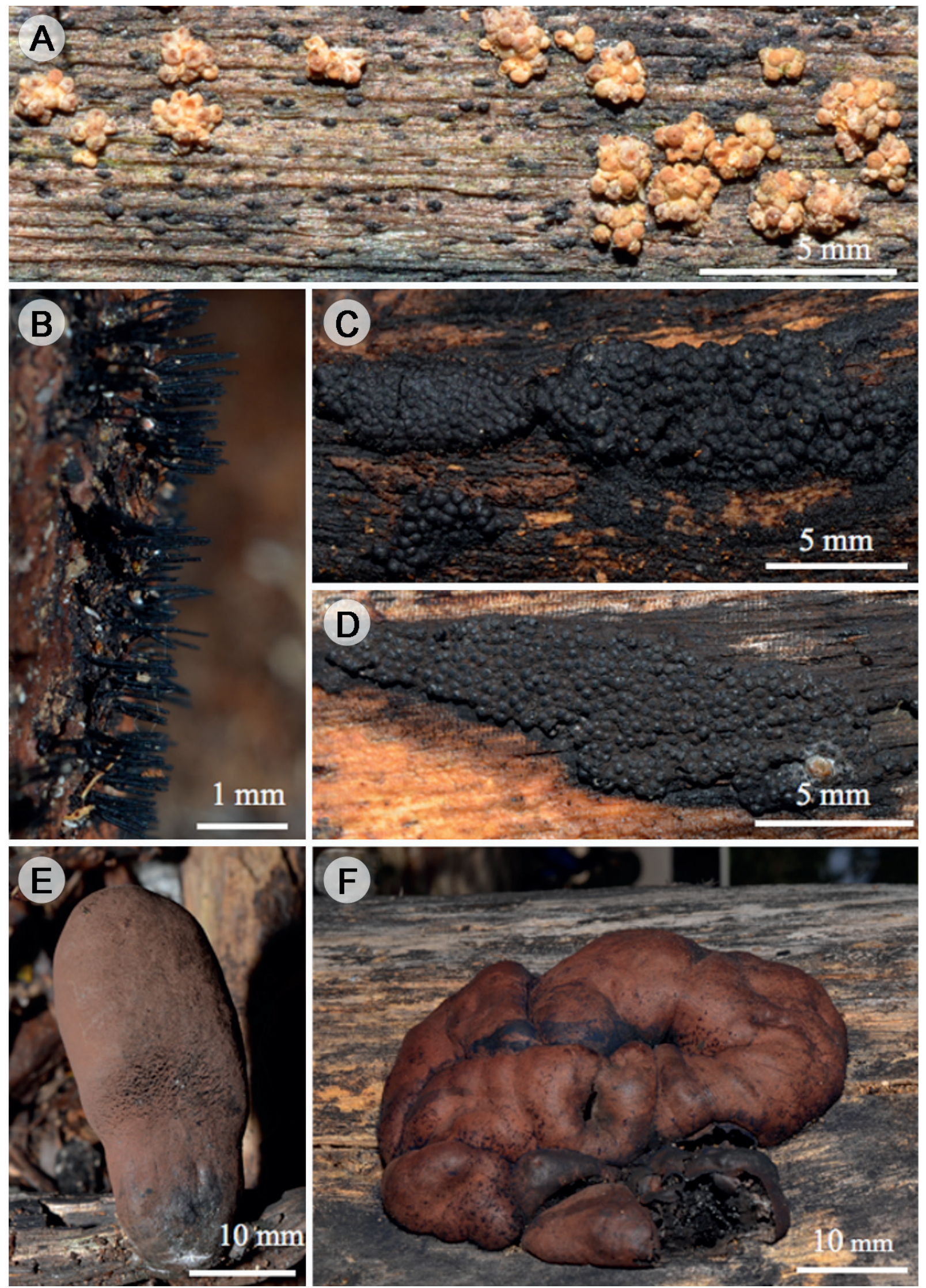

Figura 6: Sordariomycetes: A. Bionectria ochroleuca (Schwein.) Schroers \& Samuels; B. Peroneutypa scoparia (Schwein.) Carmarán \& A.I. Romero; C. Annulohypoxylon nitens (Ces.) Y.M. Ju, J.D. Rogers \& H.M. Hsieh; D. A. purpureonitens (Y.M. Ju \& J.D. Rogers) Y.M. Ju, J.D. Rogers \& H.M. Hsieh; E. Daldinia cuprea Starbäck; F. D. eschscholtzii (Ehrenb.) Rehm. 
TIPO: CUBA. En corteza, C. Wright 517 (holotipo: K).

三 Nectria macrostoma Berk. \& M.A. Curtis, in Berkeley, J. Linn. Soc., Bot. 10(46): 378. 1869.

= Hypocrea corticioides Berk. \& Broome, J. Linn. Soc., Bot. 14(74): 111. 1875.

इ Lasionectria macrostroma (Berk. \& M.A. Curtis) Cooke, Grevillea 12(64): 112. 1884.

इ Cucurbitaria macrostoma (Berk. \& M.A. Curtis) Kuntze, Revis. Gen. Pl. (Leipzig) 3(3): 461. 1898.

= Stilbum subiculosum Pat., Bull. Soc. mycol. Fr. 20(3): 138. 1904.

इ Stilbocrea macrostoma (Berk. \& M.A. Curtis) Höhn., Sber.

Akad. Wiss. Wien, Math.-naturw. Kl., Abt. 1 118: 1185. 1909.

= Clintoniella corticioides (Berk. \& Broome) Petch, Ann. R. bot. Gdns Peradeniya 7(2): 134. 1920.

Material examinado: MÉXICO. Quintana Roo, municipio Cozumel, Reserva de la Biosfera Isla Cozumel, Centro de Conservación y Educación Ambiental, 20.I.2018, T. Raymundo 7313 (ENCB).

Distribución en México: Oaxaca (Raymundo et al., 2014). Se cita por primera vez para Quintana Roo.

Xylariales

\section{Diatrypaceae}

Eutypella portoricensis (Petr.) Rappaz, Mycol. Helv. 2(3): 543. 1987.

TIPO: PUERTO RICO. Municipio Mayagüez, en corteza, 17.XII.1915, B. Fink 930 (holotipo: W 00268).

इ Peroneutypa portoricensis Petr., Annls mycol. 21(3-4): 306. 1923.

Material examinado: MÉXICO. Quintana Roo, municipio Cozumel, Reserva de la Biosfera Isla Cozumel, Parque Ecológico Punta Sur, 16.VI.2018, T. Raymundo 7665 (ENCB);
Zona Arqueológica de San Gervasio, Chen-tuk, 17.VI.2018, T. Raymundo 7675 (ENCB), 7680 (ENCB).

Distribución en México: Oaxaca (Raymundo et al., 2014). Se cita por primera vez para Quintana Roo.

Peroneutypa scoparia (Schwein.) Carmarán \& A.I. Romero, Fungal Diversity 23: 84. 2006. Fig. 6B.

TIPO: ESTADOS UNIDOS DE AMÉRICA. Carolina del Norte, en corteza de Juglans nigra L. (Juglandaceae), L. D. Schweinitz 101 (holotipo: PH).

三Sphaeria scoparia Schwein., Schr. naturf. Ges. Leipzig 1: 37. 1822.

E Valsa scoparia (Schwein.) M.A. Curtis, Geol. Nat. Hist. Surv. N. Carol. 3: 141. 1867.

= Valsa heteracantha Sacc., Atti Soc. Veneto-Trent. Sci. Nat. 2(1): 177. 1873.

= Eutypa heteracantha (Sacc.) Sacc., Syll. fung. (Abellini) 1: 177. 1882.

इ Eutypella scoparia (Schwein.) Ellis \& Everh., N. Amer. Pyren.: 495. 1892.

= Engizostoma heteracanthum (Sacc.) Kuntze, Revis. gen. pl. (Leipzig) 3(3): 474. 1898.

= Peroneutypa heteracantha (Sacc.) Berl., Icon. fung. (Abellini) 3(3-4): 81. 1902.

इ Peroneutypella scoparia (Schwein.) Berl., Icon. fung. (Abellini) 3(3-4): 84. 1902.

= Peroneutypella heteracantha (Sacc.) Rehm, Annls mycol. 4(5): 409. 1906.

इEutypa scoparia (Schwein.) L.C. Tiffany \& J.C. Gilman, lowa St. Coll. J. Sci. 40: 124. 1965.

Material examinado: MÉXICO. Quintana Roo, municipio Cozumel, Reserva de la Biosfera Isla Cozumel, Parque Ecológico Punta Sur, 16.VI.2018, T. Raymundo 7658 (ENCB).

Distribución en México: Morelos, Nuevo León, San Luis Potosí, Tamaulipas (Chacón, 2003), Oaxaca (Raymundo et al., 2014) y Sonora (Raymundo et al., 2017a). Se cita por primera vez para Quintana Roo. 


\section{Graphostromataceae}

Biscogniauxia capnodes (Berk.) Y.M. Ju \& J.D. Rogers, in Ju, Rogers, San Martín \& Granmo, Mycotaxon 66: 23. 1998.

TIPO: AUSTRALIA. Swan River, en madera descortezada, Drummond 218 (holotipo: K).

ESphaeria capnodes Berk., London J. Bot. 4: 72. 1845.

= Sphaeria pachyloma Lév. (as 'pachiloma'), Annals Sci. Nat., Bot., sér. 3 5: 259. 1846.

= Diatrype rumpens Cooke, J. Linn. Soc., Bot. 17(99): 143. 1878.

= Hypoxylon pachyloma (Lév.) Cooke, Anal. Soc. cient. argent. 12: 132. 1881.

三 Diatrype capnodes (Berk.) Sacc., Syll. fung. (Abellini) 1: 298. 1882.

= Diatrype pachyloma (Lév.) Sacc., Syll. fung. (Abellini) 1: 200. 1882.

三 Anthostoma capnodes (Berk.) Sacc., Syll. fung. (Abellini) 1: 298.1882.

= Anthostoma rumpens (Cooke) Sacc., Syll. fung. (Abellini) 1: 306. 1882.

= Nummularia australis Cooke, Grevillea 11(60): 148. 1883. = Nummularia pachyloma (Lév.) Cooke, Grevillea 12(61): 8 . 1883.

= Nummularia rumpens (Cooke) Cooke, Grevillea 12(61): 8. 1883.

三 Hypoxylon capnodes (Berk.) Berk. \& Broome ex Cooke, Grevillea 11(60): 133. 1883.

= Hypoxylon ramosum Schwein. ex Cooke, Grevillea 11(60): 132. 1883.

$=$ Nummularia mauritanica Berk. \& Cooke, in Cooke, GreviIlea 12(61): 6. 1883.

= Hypoxylon intermedium Speg., Anal. Soc. cient. argent. 18(6): 274. 1884.

= Hypoxylon pseudopachyloma Speg., Boln Acad. nac. Cienc. Córdoba 11(2): 203. 1888.

= Biscogniauxia australis (Cooke) Kuntze, Revis. gen. pl. (Leipzig) 2: 398. 1891.

= Biscogniauxia rumpens (Cooke) Kuntze, Revis. gen. pl. (Leipzig) 2: 398. 1891.

= Nummularia lataniaecola Rehm \& Bref., in Brefeld, Un- ters. Gesammtgeb. Mykol. (Liepzig) 10: 255. 1891.

= Biscogniauxia nummularia var. merrillii (Bres.) Van der Gucht, Mycotaxon 45: 267. 1992.

= Auerswaldia maxima Massee, Bull. Misc. Inf., Kew 169171: 166. 1901.

= Nummularia tenuis Starbäck, Ark. Bot. 5(7): 31. 1905.

= Nummularia asarcodes Theiss., Annls mycol. 6(4): 349. 1908.

$=$ Nummularia theissenii Syd. \& P. Syd., Annls mycol. 7(4): 344. 1909.

= Nummularia asarcodes f. griseoatra Theiss., Decades Fungorum Brasiliensium: 110. 1910.

= Nummularia starbaeckii Sacc. \& Traverso, Syll. fung. (Abellini) 20: 203. 1911.

= Nummularia maxima (Massee) Theiss. \& Syd., Annls mycol. 12(2): 179. 1914.

= Nummularia merrillii Bres., Hedwigia 56(4, 5): 307. 1915.

= Nummularia lianae Rehm, Leafl. of Philipp. Bot. 8(118): 2959. 1916.

= Nummularia alabatensis H.S. Yates, Philipp. J. Sci., C, Bot. 12(6): 378. 1917.

= Hypoxylon porteri Speg., Boln Acad. nac. Cienc. Córdoba 25: 54. 1921.

= Hypoxylon rumpens (Cooke) J.H. Mill. ex Teng, Contr. Biol. Lab. Sci. Soc. China, Bot. Ser. 7: 98. 1932.

= Hypoxylon asarcodes (Theiss.) J.H. Mill. ex Teng, Contr. to Our Knowledge of the Higher Fungi of China: 127. 1939.

= Hypoxylon nummularium var. merrillii (Bres.) J.H. Mill. ex Dennis, Kew Bull. 14(3): 450. 1960.

= Hypoxylon nummularium var. australe (Cooke) J.H. Mill., Śluzowce monogr. (Paryz): 123. 1961.

= Hypoxylon nummularium var. pseudopachyloma (Speg.) J.H. Mill., Monogr. World spec. Hypoxylon: 125. 1961.

= Hypoxylon nummularium var. rumpens (Cooke) J.H. Mill., Monogr. World spec. Hypoxylon: 123. 1961.

= Hypoxylon nummularium var. theissenii (Syd. \& P. Syd.) J.H. Mill., Monogr. World spec. Hypoxylon: 124. 1961.

= Numulariola australis (Cooke) P.M.D. Martin, Jl S. Afr. Bot. 35: 293. 1969.

= Numulariola merrillii (Bres.) P.M.D. Martin, JI S. Afr. Bot. 35: 303. 1969.

= Numulariola pseudopachyloma (Speg.) P.M.D. Martin, J S. Afr. Bot. 35: 306. 1969. 
$=$ Numulariola rumpens (Cooke) P.M.D. Martin, JI S. Afr. Bot. 35: 293. 1969.

= Numulariola theissenii (Syd. \& P. Syd.) P.M.D. Martin, JI S. Afr. Bot. 35: 293. 1969.

= Numulariola australis (Cooke) P.M.D. Martin, JI S. Afr. Bot. 42(1): 76. 1976.

$=$ Numulariola rumpens (Cooke) P.M.D. Martin, JI S. Afr. Bot. 42(1): 78. 1976.

= Numulariola theissenii (Syd. \& P. Syd.) P.M.D. Martin, JI S. Afr. Bot. 42(1): 79. 1976.

= Biscogniauxia nummularia var. pseudopachyloma (Speg.)

Whalley, in Whalley, Hywel-Jones, Jones \& Whalley, Sydowia 47(1): 73. 1995.

= Biscogniauxia capnodes var. limonispora Y.M. Ju \& J.D. Rogers (as 'limoniispora'), in Ju, Rogers, San Martín \& Granmo, Mycotaxon 66: 26. 1998.

= Biscogniauxia capnodes var. rumpens (Cooke) Y.M. Ju \& J.D. Rogers, in Ju, Rogers, San Martín \& Granmo, Mycotaxon 66: 27. 1998.

= Biscogniauxia capnodes var. theissenii (Syd. \& P. Syd.) Y.M. Ju \& J.D. Rogers, in Ju, Rogers, San Martín \& Granmo, Mycotaxon 66: 28. 1998.

Material examinado: MÉXICO. Quintana Roo, municipio Cozumel, Reserva de la Biosfera Isla Cozumel, Zona Arqueológica de San Gervasio, Chen-tuk, 17.VI.2018, T. Raymundo 7692 (ENCB).

Distribución en México: Quintana Roo (San Martín y Rogers, 1993 como Biscogniauxia nummularia var. merriIlii), Nuevo León, Tamaulipas (Ju y Rogers, 1996) y Oaxaca (Raymundo et al., 2014). Se cita por primera vez para la Isla Cozumel.

Camillea coroniformis J.D. Rogers, F. San Martín \& Y.M. Ju, Sydowia 54(1): 87. 2002.

TIPO: COSTA RICA. Lomas de Barbudal, en madera muerta de Citrus sp., 14.VII.2001, F. San Martin y J. D. Rogers 23 (holotipo: INB 3370608).

Material examinado: MÉXICO. Quintana Roo, municipio Cozumel, Reserva de la Biosfera Isla Cozumel, Centro de Conservación y Educación Ambiental, 20.I.2018, T. Raymundo 7297 (ENCB).

Distribución en México: Oaxaca (Raymundo et al., 2014). Se cita por primera vez para Quintana Roo.

\section{Hypoxylaceae}

Annulohypoxylon nitens (Ces.) Y.M. Ju, J.D. Rogers \& H.M. Hsieh, Mycologia 97(4): 861. 2005. Fig. 6C.

TIPO: MALASIA. Borneo, Sarawak, sobre corteza, Beccari 10 (holotipo: RO).

$\equiv$ Rosellinia nitens Ces., Atti Accad. Sci. fis. mat. Napoli 5(21): 13.1872.

= Hypoxylon chalybaeum Berk. \& Broome, J. Linn. Soc., Bot. 14(74): 121. 1875.

= Hypoxylon chalybaeum var. congestum Ces. (as 'congesta'), Atti Accad. Sci. fis. mat. Napoli 8(3): 17. 1879.

= Hypoxylon chalybaeum var. minor Ces., Atti Accad. Sci. fis. mat. Napoli 8(3): 17. 1879.

इ Hypoxylon nitens (Ces.) Y.M. Ju \& J.D. Rogers, Mycol. Mem. 20: 220. 1996.

Material examinado: MÉXICO. Quintana Roo, municipio Cozumel, Reserva de la Biosfera Isla Cozumel, Centro de Conservación y Educación Ambiental, 20.I.2018, A. CobosVillagrán 923 (ENCB), 924 (ENCB), 926 (ENCB); loc. cit., $T$. Raymundo 7303 (ENCB), 7311 (ENCB); loc cit., R. Valenzuela 17943 (ENCB), 17962 (ENCB); Zona Arqueológica de San Gervasio, Chen-tuk, 21.I.2018, T. Raymundo 7328 (ENCB), 7329 (ENCB), 7333 (ENCB); loc. cit., R. Valenzuela 17983 (ENCB), 18002 (ENCB), 18016 (ENCB).

Distribución en México: Chiapas, Tamaulipas (San Martín et al., 1999), Oaxaca (Raymundo et al., 2014) y Quintana Roo (García-Martínez et al., 2021b).

Annulohypoxylon purpureonitens (Y.M. Ju \& J.D. Rogers) Y.M. Ju, J.D. Rogers \& H.M. Hsieh, Mycologia 97(4): 861. 2005. Fig. 6D. 
TIPO: BRASIL. Sierra Araca, 10.III.1984, sobre corteza, G. J. Samuels 808 (holotipo: NY).

$\equiv$ Hypoxylon purpureonitens Y.M. Ju \& J.D. Rogers, Mycol. Mem. 20: 224. 1996.

Material examinado: MÉXICO. Quintana Roo, municipio Cozumel, Reserva de la Biosfera Isla Cozumel, Centro de Conservación y Educación Ambiental, 20.I.2018, T. Raymundo 7310 (ENCB); Zona Arqueológica de San Gervasio, Chen-tuk, 21.I.2018, T. Raymundo 7330 (ENCB).

Distribución en México: Chiapas (San Martín et al., 1999). Se cita por primera vez para Quintana Roo.

Daldinia cuprea Starbäck, Bih. K. svenska VetenskAkad. Handl., Afd. 3 27(9): 5. 1901. Fig. 6E.

TIPO: PARAGUAY. Parauari, Cerro Negro, 8.VIII.1893, G. A. Malme MBT177383 (lectotipo: S-F11857, isolectotipo: S-F11856).

= Daldinia granulosa Speg., Anal. Mus. nac. B. Aires 19: 345. 1909.

Material examinado: MÉXICO. Quintana Roo, municipio Cozumel, Reserva de la Biosfera Isla Cozumel, Centro de Conservación y Educación Ambiental, 20.I.2018, R. Valenzuela 17979 (ENCB).

Distribución en México: Durango, Nuevo León, Oaxaca, Querétaro, Quintana Roo, San Luis Potosí, Sinaloa, Sonora, Tabasco y Veracruz (Barbosa-Resendiz et al., 2020). Se cita por primera vez para la Isla de Cozumel.

Daldinia eschscholtzii (Ehrenb.) Rehm, Ann. Mycol. 2(2): 175. 1904. Fig. 6F.

TIPO: FILIPINAS. Isla Luzon, latitud $14.5^{\circ}$, cerca de la base del tronco, espécimen no existente (lectotipo: fig. VIII en Ehrenberg, 1820). FILIPINAS. La Laguna Prov., Isla Luzon, Mt. Makiling Peak, 1.IV.2001, T. H. Quimio y M. M. Baldovino s.n. (epitipo: K; ex CALP 11206; GenBank: HE590883).
इSphaeria eschscholtzii Ehrenb., Fung. Chamisso Coll., pl. 18, fig. 8. 1820.

इSphaeria concentrica var. eschscholtzii (Ehrenb.) Fr. Syst. mycol. 2(2):331. 1823

= Daldinia luzonensis Rehm, Philipp. J. Sci. Bot. 8: 260. 1913.

Material examinado: MÉXICO. Quintana Roo, municipio Cozumel, Zona Arqueológica de San Gervasio, Chentuk, 21.I.2018, A. Cobos-Villagrán 934 (ENCB); Reserva de la Biosfera Isla Cozumel, Centro de Conservación y Educación Ambiental, 20.I.2018, T. Raymundo 7284 (ENCB); loc. cit., R. Valenzuela 17980 (ENCB).

Distribución en México: Campeche, Chiapas, Colima, Estado de México, Guerrero, Jalisco, Morelos, Nayarit, Nuevo León, Oaxaca, Quintana Roo, San Luis Potosí, Sinaloa, Sonora, Tabasco, Tamaulipas, Veracruz y Zacatecas (BarbosaReséndiz et al., 2020).

Hypoxylon cinnabarinum (Henn.) Henn., Ann. Mus. Congo Belge, Bot., Sér. 5 2(1): 6. 1907. Fig. 7 A.

TIPO: BRASIL. Santa Catarina, prov. de Blumenau, sobre corteza, s.f., A. Möller s.n. (lectotipo: S).

इ Nummularia cinnabarina Henn., Hedwigia 36(4): 227. 1897.

= Hypoxylon rubiginosum var. tropicum J.H. Mill., Monogr. World spec. Hypoxylon: 31. 1961.

Material examinado: MÉXICO. Quintana Roo, municipio Cozumel, Reserva de la Biosfera Isla Cozumel, Centro de Conservación y Educación Ambiental, 20.I.2018, R. Valenzuela 17294 (ENCB); Zona Arqueológica de San Gervasio, Chen-tuk, 19.I.2018, R. Valenzuela 17944 (ENCB).

Distribución en México: Tabasco (Ju y Rogers, 1996), Jalisco (Guzmán-Dávalos et al., 1983), Quintana Roo, Sonora y Tamaulipas (Reyes et al., 2020).

Hypoxylon duranii Rogers, Mycotaxon 23: 429. 1985. Fig. 7B. 

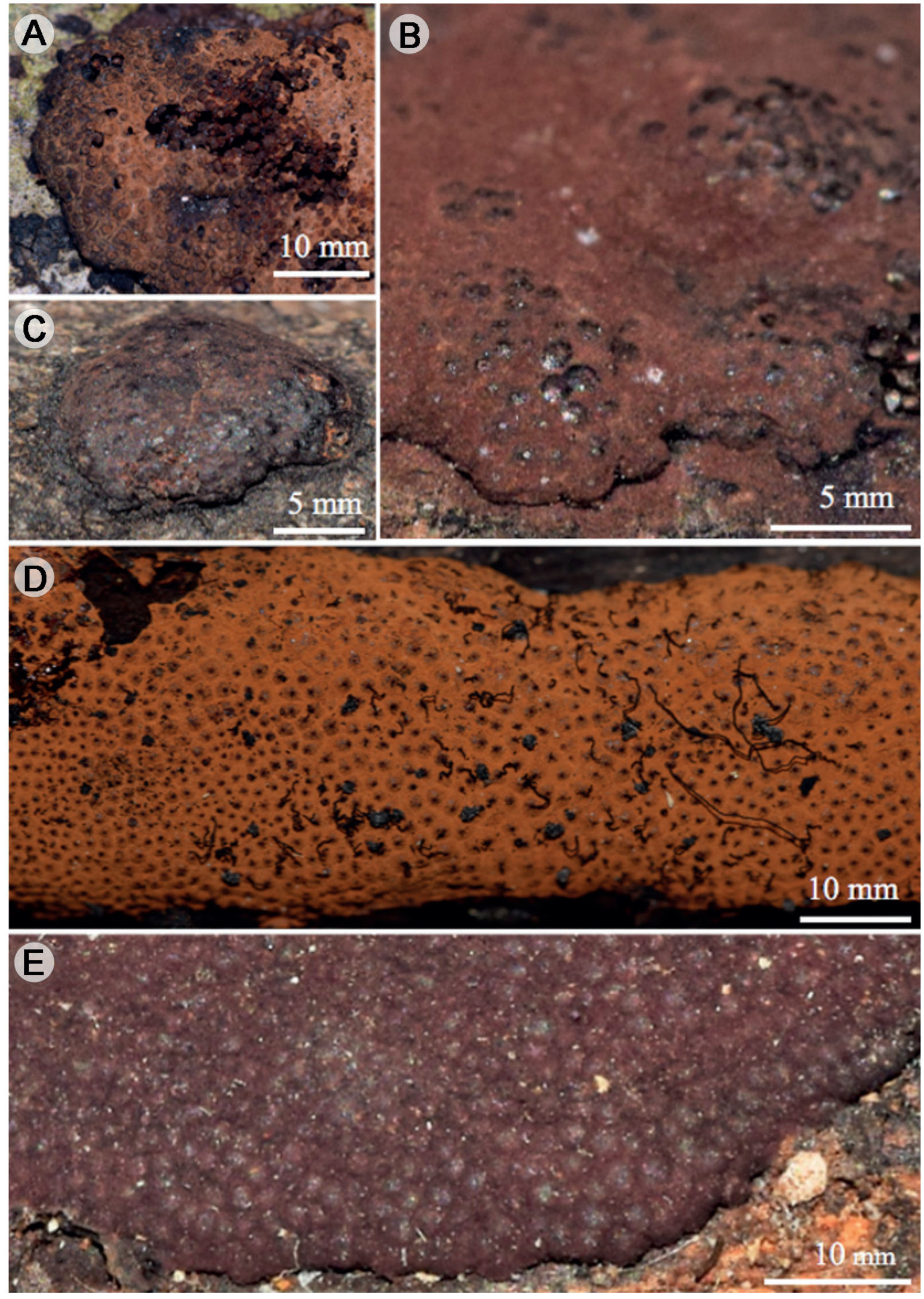

Figura 7: Sordariomycetes: A. Hypoxylon cinnabarinum (Henn.) Henn.; B. H. duranii Rogers; C. H. erythrostroma J.H. Mill.; D. H. haematostroma Mont.; E. H. investiens (Schwein.) M.A. Curtis. 
TIPO: MÉXICO. Península de Yucatán, Yucatán, ruinas de Chichén Itzá, cerca de la Autopista 180, sobre madera de leguminosa, XI.1984, R. Duran s.n. (holotipo: WSP 67597).

Material examinado: MÉXICO. Quintana Roo, municipio Cozumel, Reserva de la Biosfera Isla Cozumel, Centro de Conservación y Educación Ambiental, 20.I.2018, P. Reyes 140 (ENCB).

Distribución en México: Yucatán (Rogers, 1985) y Quintana Roo (San Martín et al., 1999). Se registra por primera vez para la Isla Cozumel.

Hypoxylon erythrostroma J.H. Mill., Mycologia 25(4): 323. 1933. Fig. 7C.

TIPO: ESTADOS UNIDOS DE AMÉRICA. Florida, Daytona, sobre árbol deciduo, I.1898, $R$. Thaxter 2 (holotipo: FLAS; isotipo: GAM2374).

= Hypoxylon gillesii J.D. Rogers \& Cand., Mycotaxon 15: 507. 1982.

Material examinado: MÉXICO. Quintana Roo, municipio Cozumel, Reserva de la Biosfera Isla Cozumel, Centro de Conservación y Educación Ambiental, 20.I.2018, R. Valenzuela 17971 (ENCB).

Distribución en México: Chiapas, Quintana Roo, Tamaulipas, Veracruz (San Martín et al., 1999), Quintana Roo y Sonora (Reyes et al., 2020).

Hypoxylon haematostroma Mont., in Sagra, Annls. Sci. Nat., Bot., sér. 2 17: 124. 1842. Fig. 7D.

TIPO: CUBA. Sobre madera, s.f., R. de la Sagra s.n. (holotipo: BPI55132, isotipo: K).

= Hypoxylon vividum Berk. \& Broome, J. Linn. Soc., Bot. 14(74): 122. 1873.

= Hypoxylon haematites Lév. ex Cooke, Grevillea 11(60): 133. 1883.
= Hypoxylon veracrucis Berk. \& Cooke, Grevillea 11(60): 129. 1883.

= Hypoxylon lucidum Ellis \& Everh., Bull. Lab. Nat. Hist. lowa State Univ. 4: 72. 1896.

= Hypoxylon stjanianum Ferd. \& Winge, Bot. Tidsskr. 29: 14. 1908.

Material examinado: MÉXICO. Quintana Roo, municipio Cozumel, Reserva de la Biosfera Isla Cozumel, Centro de Conservación y Educación Ambiental, 20.1.2018, T. Raymundo 7294 (ENCB), 7325 (ENCB); loc. cit., R. Valenzuela 17294 (ENCB); Zona Arqueológica de San Gervasio, Chentuk, 19.I.2018, R. Valenzuela 17977 (ENCB), 17994 (ENCB).

Distribución en México: Campeche, Chiapas, Morelos, Oaxaca, Quintana Roo, Tamaulipas, Veracruz, Yucatán (Guzmán, 1983; Ju y Rogers, 1996; San Martín et al., 1999; Medel, 2002; Raymundo et al., 2014), Jalisco y Quintana Roo (Reyes et al., 2020).

Hypoxylon investiens (Schwein.) M.A. Curtis, Geol. Nat. Hist. Surv. N. Carol., Pt 3: 140. 1867. Fig. 7E.

TIPO: ESTADOS UNIDOS DE AMÉRICA. Pennsylvania, Northampton County, Bethlehem, sobremadera descortezada, s.f., L.D. Schweinitz 1210 (holotipo: PH). Carolina del Norte, Salem, s.f., Collins 35 (isotipo: PH).

इSphaeria investiens Schwein., Trans. Am. phil. Soc., New Series 4(2): 193. 1832.

= Hypoxylon concurrens Berk. \& M.A. Curtis, in Berkeley, Grevillea 4(31): 93. 1876.

= Rosellinia bakeri Ellis, Torreya 5: 87. 1905.

= Hypoxylon investiens f. bakeri (Ellis) J.H. Mill. ex Dennis, Kew Bull. 15: 450. 1962.

= Hypoxylon microsporum A. Pande (as 'microspora'), Bull. bot. Surv. India 15: 8. 1973.

= Hypoxylon investiens var. magnisporum Hladki \& A.I. Romero, Darwiniana 47(2): 283. 2009.

Material examinado: MÉXICO. Quintana Roo, municipio Cozumel, Reserva de la Biosfera Isla Cozumel, Centro de 
Conservación y Educación Ambiental, R. Valenzuela 17959 (ENCB); Zona Arqueológica de San Gervasio, Chen-tuk, 20.I.2018, T. Raymundo 7295 (ENCB); loc. cit., P. Reyes 148 (ENCB); loc. cit., 17.VI.2018, R. Valenzuela 18420 (ENCB).

Distribución en México: Tabasco, Tamaulipas, Veracruz (San Martín et al., 1999; Ávalos-Lázaro et al., 2018), Quintana Roo y Sonora (Reyes et al., 2020).

Hypoxylon jaklitschii Sir \& Kuhnert, Fungal Diversity 71: 173. 2015. Fig. 8A.

TIPO: SRI LANKA. Central Province, Sigiriya, sobre madera de árbol de hoja ancha, 16.I.2013, W. Jaklitsch JF13037 (holotipo: MUCL, ex-type cultures CBS138916 \& MUCL55383; GenBank Acc. No: ITS-KM610290, ß-tubulin-KM610304).

Material examinado: MÉXICO. Quintana Roo, municipio Cozumel, Reserva de la Biosfera Isla Cozumel, Zona Arqueológica de San Gervasio, Chen-tuk, 21.I.2018, R. Valenzuela 17982 (ENCB).

Distribución en México: Jalisco, Quintana Roo y Tamaulipas (Reyes et al., 2020).

Hypoxylon lenormandii Berk. \& M.A. Curtis, J. Linn. Soc., Bot. 10(46): 385. 1868. Fig. 8B.

TIPO: CUBA. Sobre madera descortezada, s.f., C. Wright 486 (holotipo: K).

= Sphaeria subaenea Berk. \& M. A. Curtis apud Berk., J. Linn. Soc., Bot. 10: 387. 360. 1869.

= Hypoxylon oodes Berk. \& Broome, J. Linn. Soc. Bot. 14: 122. 1873.

= Rosellinia subaenea (Berk. \& M. A. Curtis) Sacc., Syll. Fung. I, p. 256. 1882.

= Hypoxylon subvinosum Speg., Anal. Soc. Cient. Argent. 18: 269. 1884.

= Hypoxylon subaeneum (Berk. \& M. A. Curtis) Speg., Anal. Soc. Cient. Argent. 26: 32. 1888.

$=$ Rosellinia melaleuca Ellis \& Everh. Apud C. L. Smith, Bull. Lowa Univ. Lab. Nat. Hist. 2: 402. 1893.
= Kretzschmaria stilbophora Rehm, Ann. Mycol. 5: 526.1907. = Rosellinia metachroa Ferd. \& Winge, Bot. Tidsskr. 29: 16. 1908.

= Hypoxylon riograndense Rehm, Ann. Mycol. 7: 154. 1909.

= Rosellinia pulchella Syd. \& P. Syd. apud Wildeman, FI.

Bas-Moyen-Congo III: 7. 1909.

= Hypoxylon fulvoochraceum Rehm, Philipp. J. Sci. 8. 188. 1913.

Material examinado: MÉXICO. Quintana Roo, municipio Cozumel, Reserva de la Biosfera Isla Cozumel, Centro de Conservación y Educación Ambiental, 20.I.2018, R. Valenzuela 17957 (ENCB), 18017 (ENCB); Parque Ecológico Punta Sur, 16.VI.2018, T. Raymundo 7656 (ENCB); loc. cit., R. Valenzuela 18359 (ENCB); loc. cit., 14.X.2019, T. Raymundo 8319 (ENCB); Zona Arqueológica de San Gervasio, Chentuk, R. Valenzuela 17790 (ENCB).

Distribución en México: Campeche, Chiapas, Jalisco, Nayarit, Nuevo León, Oaxaca, Quintana Roo, Sinaloa, Sonora, Tamaulipas y Yucatán (Martin, 1969; Ju y Rogers, 1996; San Martín et al., 1999; Medel, 2002; Raymundo et al., 2014, 2017a; Reyes et al., 2020).

Hypoxylon perforatum (Schwein.) Fr., Summa veg. Scand., Sectio Post.: 384. 1849. Fig. 8C.

TIPO: ESTADOS UNIDOS DE AMÉRICA. Pennsylvania, Northampton County, Bethlehem, sobre corteza, s.f., L. D. Schweinitz 1194 (lectotipo: PH, designado por Ju y Rogers, 1996).

इ Sphaeria perforata Schwein., Schr. naturf. Ges. Leipzig 1:31. 1822.

= Sphaeria durissima Schwein., Schr. naturf. Ges. Leipzig 1:32. 1822.

= Sphaeria catalpa Schwein., Trans. Am. phil. Soc., New Series 4(2): 193. 1832.

= Sphaeria decorticata Schwein., Trans. Am. phil. Soc., New Series 4(2): 191. 1832.

= Sphaeria leucostigma Lév., Annls Sci. Nat., Bot., sér. 3 9:142. 1848.

= Hypoxylon decorticatum M.A. Curtis, Geol. Nat. Hist. Surv. N. Carol., Pt 3: 140. 1867. 

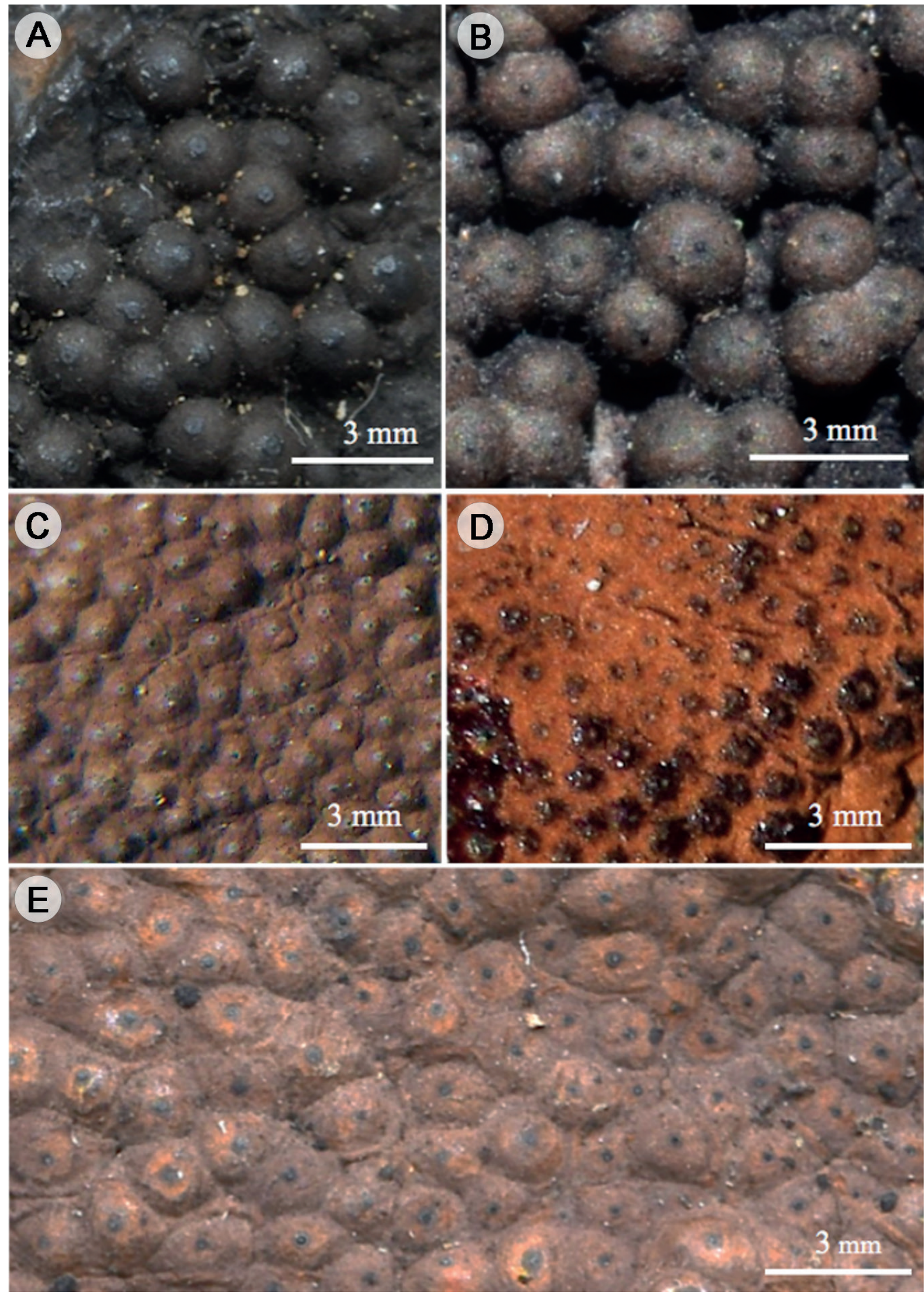

Figura 8: Sordariomycetes: A. Hypoxylon jaklitschii Sir \& Kuhnert; B. H. lenormandii Berk. \& M.A. Curtis; C. H. perforatum (Schwein.) Fr. D. H. rickii Y.M. Ju \& J.D. Rogers; E. H. subgilvum Berk. \& Broome 
= Hypoxylon catalpa (Schwein.) Sacc., Syll. fung. (Abellini) 1:392. 1882.

= Hypoxylon durissimum (Schwein.) Sacc., Syll. fung. (Abellini) 1: 378. 1882.

= Hypoxylon leucostigma (Lev.) Sacc., Syll. fung. (Abellini) 1:367. 1882.

= Hypoxylon luridum Nitschke, Pyrenomyc. Germ. 1: 31.1867.

= Hypoxylon plumbeum Speg., Anal. Soc. Cient. Argent.18(6): 270. 1884.

= Hypoxylon rubiginosum var. microcarpum Speg., Anales Mus. Nac. B. Aires, Ser. 3 17(10): 120. 1908.

इ Hypoxylon rubiginosum var. perforatum (Schwein.) L.E. Petrini, Mycol. Helv. 1(7): 531. 1986.

Material examinado: MÉXICO. Quintana Roo, municipio Cozumel, Reserva de la Biosfera Isla de Cozumel, Centro de Conservación y Educación Ambiental, 20.I.2018, P. Reyes 141 (ENCB), 146 (ENCB); loc. cit., R. Valenzuela 17942 (ENCB); Zona Arqueológica de San Gervasio, Chen-tuk, 19.I.2018, P. Reyes 139 (ENCB).

Distribución en México: Nuevo León (San Martín et al., 1999), Jalisco y Quintana Roo (Reyes et al., 2020).

Hypoxylon rickii Y.M. Ju \& J.D. Rogers, Mycol. Mem. 20: 174. 1996. Fig. 8D.

TIPO: BRASIL. Sobre madera descortezada, s.f., J. Rick 303 (holotipo: NY).

Material examinado: MÉXICO. Quintana Roo, municipio Cozumel, Reserva de la Biosfera Isla Cozumel, Centro de Conservación y Educación Ambiental, 20.I.2018, $R$. Valenzuela 18012 (ENCB); Zona Arqueológica de San Gervasio, Chen-tuk, 21.I.2018, P. Reyes 149 (ENCB); loc. cit., 19.I.2018, R. Valenzuela 17970 (ENCB).

Distribución en México: Oaxaca (San Martín et al., 1999), Quintana Roo, Sonora y Tamaulipas (Reyes et al., 2020).

Hypoxylon subgilvum Berk. \& Broome, J. Linn. Soc., Bot. 14(74): 120. 1873. Fig. 8E.
TIPO: SRI LANKA. Central Province, sobre corteza, XII.1868, G. H. K. Thwaite 1087 (holotipo: K).

= Hypoxylon caaguazu Speg., Anal. Soc. Cient. Argent. 18(6): 275.1884.

= Hypoxylon hypomiltum var. microsporum Y. Abe, Trans. Mycol. Soc. Japan 27: 55. 1986.

= Hypoxylon subgilvum var. microsporum (Y. Abe) Y.M. Ju \& J.D. Rogers, Mycol. Mem. 20: 188. 1996.

Material examinado: MÉXICO. Quintana Roo, municipio Cozumel, Reserva de la Biosfera Isla Cozumel, Centro de Conservación y Educación Ambiental, 20.I.2018, T. Raymundo 7307 (ENCB); Zona Arqueológica de San Gervasio, Chen-tuk, 19.I.2018, T. Raymundo 7334 (ENCB); loc. cit., $R$. Valenzuela 18011 (ENCB).

Distribución en México: Campeche, Tamaulipas (San Martín et al., 1999), Jalisco y Quintana Roo (Reyes et al., 2020; García-Martínez et al., 2021b).

Hypoxylon sublimbatum (Durieu \& Mont.) P. M. D. Martin, JI S. Afr. Bot. 42(1): 74. 1976.

TIPO: ARGELIA. Cerca de Argel, en columna de Ampelodesmos mauritanicus (Poir.) T. Durand \& Schinz, Durieu \& Montagne 36 (holotipo: $\mathrm{K}$ ).

इSphaeria sublimbata Durieu \& Mont., in Durieu, Expl. Sci. Alg., Fl. Algér. 1(13): 498. 1849.

इ Rosellinia sublimbata (Durieu \& Mont.) Pass., in Thümen, Inst. Coimbra 294: 33. 1879.

इ Anthostomella sublimbata (Durieu \& Mont.) Speg., Revta Fac. Agron. Vet. Univ. nac. La Plata, Ser. 2 6(1): 38. 1910.

$\equiv$ Astrocystis sublimbata (Durieu \& Mont.) G.C. Hughes, Mycol. Pap. 50: 9. 1953.

इ Hypoxylon sublimbatum (Durieu \& Mont.) P.M.D. Martin, JI S. Afr. Bot. 33: 322. 1967.

Material examinado: MÉXICO. Quintana Roo, municipio Cozumel, Reserva de la Biosfera Isla de Cozumel, Parque Ecológico Punta Sur, 16.VI.2018, R. Valenzuela (ENCB). 
Distribución en México: Chiapas, Quintana Roo y Tamaulipas (San Martín y Rogers, 1995). Se cita por primera vez para la Isla Cozumel.

Jackrogersella multiformis (Fr.) L. Wendt, Kuhnert \& M. Stadler, Mycol. Prog. 14(1): 24. 2018.

TIPO: SUECIA. Localidad desconocida, sobre madera, E. Fries 44 (holotipo: UPS, isotipo: K).

= Hypoxylon granulosum Bull., Hist. Champ. Fr. (Paris) 1(1): 176. 1791.

= Sphaeria crustacea Sowerby, Col. fig. Engl. Fung. Mushr. (Londres) 3(25): 372. 1802.

= Sphaeria cinereofusca Schumach., Enum. pl. (Kjbenhavn) 2: 164.1803.

इ Sphaeria multiformis Fr., Observ. Mycol. (Havniae) 1: 169. 1815. ALEMANIA. North Rhine Westphalia: Haan-Gruiten, sobre Alnus glutinosa (L.) Gaertn., 10.VIII.2004, M. Stadler s.n. (epitipo: STMA 04117, designado en Wendt et al. (2018). = Sphaeria multiformis var. alnea Fr., Observ. mycol. (Havniae) 1: 170. 1815.

= Sphaeria multiformis var. coarctata Fr., Observ. mycol. (Havniae) 1: 170. 1815.

= Sphaeria multiformis var. dilatata Fr., Observ. mycol. (Havniae) 1: 170. 1815.

= Nemania crustacea (Sowerby) Gray, Nat. Arr. Brit. PI. 1: 517. 1821.

= Peripherostoma granulosum (Bull.) Gray, Nat. Arr. Brit. PI. (Londres) 1: 514. 1821.

= Stromatosphaeria elliptica Grev., Fl. Edin.: 357. 1824.

= Sphaeria corrugata Fr., Elench. fung. (Greifswald) 2: 70. 1828.

इ Hypoxylon multiforme (Fr.) Fr., Summa veg. Scand., Sectio Post. (Stockholm): 384. 1849.

= Hypoxylon corrugatum (Fr.) Fr., Summa veg. Scand., Sectio Post. (Stockholm): 384. 1849.

= Hypoxylon crustaceum (Sowerby) Nitschke, Pyrenomyc. Germ. 1: 49. 1867.

= Hypoxylon multiforme var. effusum (Pers.) Cooke y Ellis, Grevillea 5(33): 33. 1876.

= Hypoxylon multiforme var. granulosum (Bull.) Sacc., Syll. fung. (Abellini) 1: 364. 1882.
= Hypoxylon hookeri Berk. ex Cooke, Grevillea 11(60): 129. 1883.

= Rosellinia callimorpha P. Karst., Hedwigia 23(6): 84. 1884.

= Hypoxylon granulosum var. luxurians Rehm, Annls mycol. 3(3): 229. 1905.

= Hypoxylon callimorphum (P. Karst.) P.M.D. Martin, JI S. Afr. Bot 33: 325.1967.

= Annulohypoxylon multiforme Y.M. Ju \& J.D. Rogers, Mycol. Mem. 20: 219. 1996.

इ Annulohypoxylon multiforme (Fr.) Y.M. Ju, J.D. Rogers \& H.M. Hsieh, Mycologia 97(4): 859. 2005.

Material estudiado: MÉXICO. Quintana Roo, municipio Cozumel, Parque Ecológico Punta Sur, 24.IV.2019, Y. A. García y M. A. Bravo 59 (ENCB), 54 (ENCB); loc.cit., 16.X.2019, T. Raymundo 8328 (ENCB).

Distribución en México: Tamaulipas (San Martín et al., 1999). Se cita por primera vez para Quintana Roo.

\section{Xylariaceae}

Kretzschmaria zonata (Lév.) P.M.D. Martin, JI S. Afr. Bot. 42(1): 75. 1976.

TIPO: INDONESIA. Java, en palmas Zippelius, M. J. H. Léveillé 253 (holotipo: MNHN).

三Sphaeria zonata Lév., Annls Sci. Nat., Bot., sér. 3 (3): 48. 1845.

三 Ustulina zonata (Lév.) Sacc., Syll. fung. (Abellini) 1: 352. 1882.

= Ustulina vulgaris $\mathrm{f}$. madagascariensis Henn., in Voeltzkow, Reise in Ost-Africa 1903-1905, Syst. Arbeit. 3(1-3): 30. 1908. = Hypoxylon deustum f. madagascariense.(Henn.) Hendr. (as 'madagascariensis'), Publ. Inst. Natl. Agron. Congo Belge. 35: 7. 1948.

Material examinado: MÉXICO. Quintana Roo, municipio Cozumel, Reserva de la Biosfera Isla Cozumel, Zona Arqueológica de San Gervasio, Chen-tuk, 17.VI.2018, T. Raymundo 7689 (ENCB). 
Distribución en México: se ha citado de Campeche, Chiapas, Nuevo León, Quintana Roo, Tabasco, Tamaulipas y Veracruz (San Martín y Lavin, 1997; Cibrián-Tovar, 2013). Se registra por primera vez para la Isla Cozumel.

Rosellinia subiculata (Schwein.) Sacc., Syll. fung. (Abellini) 1: 255. 1882.

TIPO: ESTADOS UNIDOS DE AMÉRICA. Carolina del Norte, en madera de Liriodendron y Quercus tomentosa, L. D. Schweinitz 175 (no está indicado el tipo).

इSphaeria subiculata Schwein., Schr. naturf. Ges. Leipzig 1: 44. 1822.

Material examinado: MÉXICO. Quintana Roo, municipio Cozumel, Reserva de la Biosfera Isla Cozumel, Zona Arqueológica de San Gervasio, Chen-tuk, 21.I.2018, T. Raymundo 7320 (ENCB), 7335 (ENCB); loc. cit., R. Valenzuela 17996 (ENCB).

Distribución en México: Hidalgo (Raymundo et al., 2016) y Veracruz (San Martín y Rogers, 1995). Se cita por primera vez para Quintana Roo.

Xylaria bambooensis Lloyd, Mycol. Writ. 7(72): 1285. 1924.

TIPO: HONDURAS. En Bambusaceae, no está indicado el tipo.

Material examinado: MÉXICO. Quintana Roo, municipio Cozumel, Reserva de la Biosfera Isla Cozumel, Zona Arqueológica de San Gervasio, Chen-tuk, 21.I.2018, A. CobosVillagrán 935 (ENCB).

Distribución en México: Quintana Roo (Guzmán, 2003). Se registra por primera vez para la Isla Cozumel.

Xylaria cubensis (Mont.) Fr., Nova Acta Regiae Societatis Scientiarum Upsaliensis Ser. 3, 1: 126. 1851.

TIPO: CUBA. No está indicado el tipo.
इ Hypoxylon cubense Mont., Annls Sci. Nat., Bot., sér. 2 13: 345. 1840.

= Sphaeria flabelliformis Schwein., in Fries, Elench. fung. (Greifswald) 2: 58. 1828.

= Xylaria flabelliformis (Schwein.) Berk. \& M.A. Curtis, in Berkeley, J. Linn. Soc., Bot. 10(46): 381. 1869.

= Isaria flabelliformis (Schwein.) Lloyd, Mycol. Writ. 4(Mycol. Notes 40): 547. 1916.

= Xylosphaera cubensis (Mont.) Dennis, Kew Bull. 13(1): 103. 1958.

= Xylosphaera papyrifera subsp. cubensis (Mont.) Dennis, Bull. Jard. bot. État Brux. 31: 122. 1961.

= Xylaria papyrifera subsp. cubensis (Mont.) D. Hawksw., Trans. Br. mycol. Soc. 61(1): 200.1973.

=Xylocoremium flabelliforme (Schwein.) J.D. Rogers, Mycologia 76(5): 914. 1984.

Material examinado: MÉXICO. Quintana Roo, municipio Cozumel, Reserva de la Biosfera Isla Cozumel, Zona Arqueológica de San Gervasio, Chen-tuk, 21.I.2018, A. CobosVillagrán 933 (ENCB).

Distribución en México: Chiapas, Quintana Roo, Tamaulipas (San Martín y Rogers, 1989; Guzmán, 2003), Hidalgo (Raymundo et al., 2016) y Veracruz (Medel et al., 2008).

Se registra por primera vez para la Isla Cozumel.

Xylaria mellisii (Berk.) Cooke, Grevillea 11(59): 85. 1883.

TIPO: INGLATERRA. Isla Santa Helena, en madera, J.C. Mellis s.n. (holotipo: K).

इ Hypoxylon mellissii Berk., in Melliss, St. Helena: A Physical, Historical and Topographical Description of the Island, including the Geology, Fauna, Flora and Meteorology: 379. 1875.

इXylosphaera mellissii (Berk.) Dennis, Kew Bull. 13(1): 104. 1958.

= Xylosphaera mellissii var. nuda Dennis (as 'mellisii'), Revta Biol., Lisb. 1(3-4): 183. 1958.

= Xylaria mellissii var. nuda (Dennis) D. Hawksw., Trans. Br. mycol. Soc. 61(1): 200. 1973. 
Material examinado: MÉXICO. Quintana Roo, municipio Cozumel, Reserva de la Biosfera Isla Cozumel, Centro de Conservación y Educación Ambiental, 20.I.2018, A. CobosVillagrán 962 (ENCB).

Distribución en México: Michoacán (Becerril-Navarrete et al., 2018), Tamaulipas y Veracruz (Medel et al., 2008). Se cita por primera vez para Quintana Roo.

\section{Discusión}

Los ascomicetos de la Reserva de la Biosfera Isla Cozumel presentan una alta riqueza, pues ahí se encuentran 40 taxones distribuidos en las clases Dothideomycetes (9 spp.), Leotiomycetes (1 spp.), Pezizomycetes (2 spp.) y Sordariomycetes (28 spp.). Esta última tiene $70 \%$ de la composición total, mientras que el orden Xylariales, con 25 especies, es el grupo de hongos con mayor riqueza, lo cual coincide con De la Fuente et al. (2020), quienes publicaron el listado de Ascomycota, Basidiomycota y Glomeromycota del estado de Quintana Roo, en donde este orden es el mejor representado con 72 especies. Hypoxylaceae, con 16 taxones, se muestra como la familia más abundante con $40 \%$ del total de los ascomicetos determinados. El género Hypoxylon Bull., con 11 especies, representa $27.5 \%$ del total, le siguen Xylaria Hill ex Schrank y Rhytidhysteron Speg. con $7.5 \%$ y tres taxones cada uno.

En el Cuadro 1 se observa que el Centro de Conservación y Educación Ambiental (CCEA) y la Zona Arqueológica de San Gervasio (ZASG) presentan una mayor riqueza taxonómica con 23 y 25 especies, respectivamente. En contraste, en el Parque Ecológico Punta Sur (PEPS) se encontraron 11 , lo cual puede deberse a que la diversidad vegetal y la acumulación de materia orgánica (Hyde y Lee, 1995) en la selva baja caducifolia del CCEA y la selva mediana subcaducifolia en la ZASG es mayor que en manglares y dunas costeras del PEPS, favoreciendo asi la colonización fúngica en dichos sitios de estudio. Las especies que se encontraron en todas las localidades son Bionectria ochroleuca, Hypoxylon lenormandii, Rhytidhysteron neorufulum y $R$. rufulum. Mientras que por localidad se determinaron 23 taxones para CCEA que equivalen a $57.5 \%$ del total. Bertia tropicalis, Camillea coroniformis, Hypocreopsis macrostoma, H. duranii, H. erythrosoma, Macrovalsaria megalospo- ra y Xylaria mellisii se recolectaron únicamente en la selva baja caducifolia de esta localidad. Doce taxones que equivalen a $30 \%$ del total registrado en PEPS, de las cuales Botryopsphaeria dothidea, Eutypella portoricensis, Hypoxylon sublimbatum, Lasiodiplodia theobromae, Peroneutypa scoparia y Platystomum mexicanum, fueron exclusivos de la zona dunera. Por su parte, Patellaria atrata es la única especie que se observó sobre Rhizophora mangle. Para la ZASG se determinaron 25 especies que equivalen a $62.5 \%$ del total. De estas, Anteaglonium abbreviatum, Biscogniauxia capnodes, Gloniella tropicalis, H. jaklitschii, Jackrogersella multiformis, Kretzschmaria zonata, Rosellinia subiculata, Unguiculariopsis ravenelii, Xylaria bambooensis y $X$. cubensis se localizaron especificamente en selva mediana subcaducifolia de dicha localidad. Por su parte, Annulohypoxylon nitens, A. purpureonitens, Cookeina tricholoma, Daldinia eschscholtzii, $H$. haematostroma, $H$. investiens, $H$. rickii, H. subgilvum y Phillipsia domingensis fueron localizados tanto en CCEA como en ZASG. Las especies con mayor número de ejemplares fueron Rhytidhysteron rufulum con 14 y Annulohypoxylon nitens con 13.

En cuanto al hábito nutricional de los hongos recolectados, 34 especies (85\%) son saprobias; y cinco (12.5\%) son fitopatógenas, porque se encontraron sobre árboles vivos y están representadas por Bionectria ochroleuca, Botryosphaeria dothidea, Krtezschmaria zonata, Lasiodiplodia theobromae y Macrovalsaria megalospora. Unguiculariopsis ravenelii fue la única especie micoparásita y se determinó creciendo sobre Rhytidhysteron rufulum.

A todas las especies de ascomicetos estudiados se les pudieron determinar los hospederos, destacando 20 especies de plantas y solo una de hongo. La familia Fabaceae es la que presentó el mayor número de taxa de hongos con 15, siguiéndole la familia Burseraceae con ocho, y las familias Meliaceae y Moraceae con siete. De los hospederos vegetales sobresalen Brosimum alicastrum, Bursera simaruba, Cedrela odorata y Enterolobium cyclocarpum (Jacq.) Griseb. por tener siete u ocho especies de hongos creciendo en ellas. Anteaglonium parvulum, Bionectria ochroleu$\mathrm{ca}$, Eutypella portoricensis, Hypoxylon haematostroma, $\mathrm{H}$. lenormandii, $H$. perforatum, $H$. subgilvum y Peroneutypa scoparia crecen sobre Enterolobium cyclocarpum. Bertia tropicalis, Biscogniauxia capnodes, Daldinia eschscholtzii, 
Cuadro 1: Ascomicetos de la Reserva de la Biosfera Isla Cozumel, Quintana Roo, México.

\begin{tabular}{|c|c|c|c|c|c|c|}
\hline Especie & $\begin{array}{l}\text { Centro de } \\
\text { Conservación } \\
\text { y Educación } \\
\text { Ambiental }\end{array}$ & $\begin{array}{l}\text { Parque } \\
\text { Ecológico } \\
\text { Punta Sur }\end{array}$ & $\begin{array}{l}\text { Zona Arqueológica } \\
\text { San Gervasio, } \\
\text { Chen-tuk }\end{array}$ & $\begin{array}{l}\text { Hospedero: } \\
\text { Nombre científico } \\
\text { Nombre común }\end{array}$ & Familia & Hábito \\
\hline $\begin{array}{l}\text { Annulohypoxylon nitens } \\
\text { (Ces.) Y.M. Ju, J.D. Rogers \& }\end{array}$ & $x$ & & $x$ & $\begin{array}{l}\text { Guazuma ulmifolia Lam. } \\
\text { Piixoy }\end{array}$ & Malvaceae & saprobio \\
\hline H. M. Hsieh & & & & $\begin{array}{l}\text { Mimosa bahamensis Benth. } \\
\text { Motita } \\
\text { Senna racemosa (Mill.) H.S. Irwin } \\
\text { \& Barneby } \\
\text { Ja' abin }\end{array}$ & Fabaceae & \\
\hline $\begin{array}{l}\text { Annulohypoxylon } \\
\text { purpureonitens (Y.M. Ju \& } \\
\text { J.D. Rogers) Y.M. Ju, J.D. } \\
\text { Rogers \& H.M. Hsieh }\end{array}$ & $x$ & & $x$ & $\begin{array}{l}\text { Mimosa bahamensis Benth. } \\
\text { Motita } \\
\text { Guazuma ulmifolia Lam. } \\
\text { Piixoy }\end{array}$ & Malvaceae & saprobio \\
\hline $\begin{array}{l}\text { Anteaglonium abbreviatum } \\
\text { (Schwein.) Mugambi \& } \\
\text { Huhndorf }\end{array}$ & & & $x$ & $\begin{array}{l}\text { Enterolobium cyclocarpum (Jacq.) } \\
\text { Griseb. } \\
\text { Piich }\end{array}$ & Fabaceae & saprobio \\
\hline $\begin{array}{l}\text { Bertia tropicalis Huhndorf, } \\
\text { A.N. Mill. \& F.A. Fernández }\end{array}$ & $x$ & & & $\begin{array}{l}\text { Cedrela odorata L. Cedro } \\
\text { Bursera simaruba (L.) Sarg. } \\
\text { Chakaa' }\end{array}$ & $\begin{array}{l}\text { Meliaceae } \\
\text { Burseraceae }\end{array}$ & saprobio \\
\hline $\begin{array}{l}\text { Bionectria ochroleuca } \\
\text { (Schwein.) Schroers \& } \\
\text { Samuels }\end{array}$ & $x$ & $x$ & $x$ & $\begin{array}{l}\text { Enterolobium cyclocarpum Griseb. } \\
\text { Piich }\end{array}$ & Fabaceae & fitopatógeno \\
\hline $\begin{array}{l}\text { Biscogniauxia capnodes } \\
\text { (Berk.) Y.M. Ju \& J.D. Rogers }\end{array}$ & & & $x$ & $\begin{array}{l}\text { Bursera simaruba (L.) Sarg. } \\
\text { Chakaa' }\end{array}$ & Burseraceae & saprobio \\
\hline $\begin{array}{l}\text { Botryosphaeria dothidea Ces } \\
\text { \& De Not. }\end{array}$ & & $x$ & & $\begin{array}{l}\text { Carica papaya L. } \\
\text { Papaya }\end{array}$ & Caricaceae & fitopatógeno \\
\hline $\begin{array}{l}\text { Camillea coroniformis J.D. } \\
\text { Rogers, F. San Martín \& } \\
\text { Y.M. Ju }\end{array}$ & $x$ & & & $\begin{array}{l}\text { Lysiloma latisiliquum (L.) Benth. } \\
\text { Tsalam }\end{array}$ & Fabaceae & saprobio \\
\hline $\begin{array}{l}\text { Cookeina tricholoma (Mont.) } \\
\text { Kuntze }\end{array}$ & $x$ & & $x$ & $\begin{array}{l}\text { Cedrela odorata L. } \\
\text { Cedro }\end{array}$ & Meliaceae & saprobio \\
\hline Daldinia cuprea Starbäck & $\mathrm{x}$ & & & $\begin{array}{l}\text { Manilkara zapota (L.) P. Royen } \\
\text { Sapotillo }\end{array}$ & Sapotaceae & saprobio \\
\hline $\begin{array}{l}\text { Daldinia eschscholtzii } \\
\text { (Ehrenb.) Rehm }\end{array}$ & $x$ & & $x$ & $\begin{array}{l}\text { Bursera simaruba (L.) Sarg. } \\
\text { Chakaa' }\end{array}$ & Burseraceae & saprobio \\
\hline $\begin{array}{l}\text { Eutypella portoricensis (Petr.) } \\
\text { Rappaz }\end{array}$ & & $\mathrm{X}$ & & $\begin{array}{l}\text { Enterolobium cyclocarpum Griseb. } \\
\text { Piich }\end{array}$ & Fabaceae & saprobio \\
\hline $\begin{array}{l}\text { Gloniella tropicalis I. Álvarez, } \\
\text { Raymundo \& R. Valenz. }\end{array}$ & & & $x$ & $\begin{array}{l}\text { Gliricidia sepium (Jacq.) Kunth ex } \\
\text { Walp. } \\
\text { Cocoite }\end{array}$ & Fabaceae & saprobio \\
\hline $\begin{array}{l}\text { Hypocreopsis macrostoma } \\
\text { (Berk. \& M.A. Curtis) E. } \\
\text { Müll. }\end{array}$ & $x$ & & & $\begin{array}{l}\text { Bambusa vulgaris Schrad. ex J.C. } \\
\text { Wendl } \\
\text { Bambú }\end{array}$ & Poaceae & saprobio \\
\hline $\begin{array}{l}\text { Hypoxylon cinnabarinum } \\
\text { (Henn.) Henn. }\end{array}$ & $x$ & & $x$ & $\begin{array}{l}\text { Brosimum alicastrum Sw. } \\
\text { Ramón }\end{array}$ & Moraceae & saprobio \\
\hline
\end{tabular}


Cuadro 1: Continuación.

\begin{tabular}{|c|c|c|c|c|c|c|}
\hline Especie & $\begin{array}{l}\text { Centro de } \\
\text { Conservación } \\
\text { y Educación } \\
\text { Ambiental }\end{array}$ & $\begin{array}{l}\text { Parque } \\
\text { Ecológico } \\
\text { Punta Sur }\end{array}$ & $\begin{array}{l}\text { Zona Arqueológica } \\
\text { San Gervasio, } \\
\text { Chen-tuk }\end{array}$ & $\begin{array}{l}\text { Hospedero: } \\
\text { Nombre científico } \\
\text { Nombre común }\end{array}$ & Familia & Hábito \\
\hline Hypoxylon duranii Rogers & $x$ & & & $\begin{array}{l}\text { Lysiloma latisiliquum (L.) Benth. } \\
\text { Tsalam }\end{array}$ & Fabaceae & saprobio \\
\hline $\begin{array}{l}\text { Hypoxylon erythrostroma } \\
\text { J.H. Mill. }\end{array}$ & $x$ & & & $\begin{array}{l}\text { Bursera simaruba (L.) Sarg. } \\
\text { Chakaa' }\end{array}$ & Burseraceae & saprobio \\
\hline \multirow[t]{5}{*}{$\begin{array}{l}\text { Hypoxylon haematostroma } \\
\text { Mont. }\end{array}$} & $x$ & & $x$ & $\begin{array}{l}\text { Cedrela odorata } \mathrm{L} . \\
\text { Cedro }\end{array}$ & Meliaceae & saprobio \\
\hline & & & & $\begin{array}{l}\text { Enterolobium cyclocarpum Griseb. } \\
\text { Piich }\end{array}$ & Fabaceae & \\
\hline & & & & $\begin{array}{l}\text { Delonix regia (Bojer ex Hook.) Raf. } \\
\text { Flamboyan }\end{array}$ & Fabaceae & \\
\hline & & & & $\begin{array}{l}\text { Bursera simaruba (L.) Sarg. } \\
\text { Chakaa' }\end{array}$ & Burseraceae & \\
\hline & & & & $\begin{array}{l}\text { Brosimum alicastrum Sw. } \\
\text { Ramón }\end{array}$ & Moraceae & \\
\hline $\begin{array}{l}\text { Hypoxylon investiens } \\
\text { (Schwein.) M.A. Curtis }\end{array}$ & $x$ & & $x$ & $\begin{array}{l}\text { Cedrela odorata } \mathrm{L} . \\
\text { Cedro }\end{array}$ & Meliaceae & saprobio \\
\hline $\begin{array}{l}\text { Hypoxylon jaklitschii Sir \& } \\
\text { Kuhnert }\end{array}$ & & & $x$ & $\begin{array}{l}\text { Brosimum alicastrum Sw. } \\
\text { Ramón }\end{array}$ & Moraceae & saprobio \\
\hline \& M.A. Curtis & $x$ & $x$ & $x$ & $\begin{array}{l}\text { Cedrela odorata, L. } \\
\text { Cedro } \\
\text { Enterolobium cyclocarpum Griseb. } \\
\text { Piich } \\
\text { Bursera simaruba (L.) Sarg. } \\
\text { Chakaa' } \\
\text { Brosimum alicastrum Sw. } \\
\text { Ramón }\end{array}$ & $\begin{array}{l}\text { Burseraceae } \\
\text { Moraceae }\end{array}$ & saprobio \\
\hline $\begin{array}{l}\text { Hypoxylon perforatum } \\
\text { (Schwein.) Fr. }\end{array}$ & $x$ & & $x$ & $\begin{array}{l}\text { Brosimum alicastrum Sw. } \\
\text { Ramón } \\
\text { Enterolobium cyclocarpum Griseb. } \\
\text { Piich }\end{array}$ & Fabaceae & saprobio \\
\hline $\begin{array}{l}\text { Hypoxylon rickii Y.M. Ju \& J.D. } \\
\text { Rogers }\end{array}$ & $x$ & & $x$ & $\begin{array}{l}\text { Cedrela odorata } \mathrm{L} . \\
\text { Cedro } \\
\text { Brosimum alicastrum Sw. } \\
\text { Ramón }\end{array}$ & Moraceae & saprobio \\
\hline \multirow[t]{3}{*}{$\begin{array}{l}\text { Hypoxylon subgilvum Berk. \& } \\
\text { Broome }\end{array}$} & $x$ & & $x$ & $\begin{array}{l}\text { Enterolobium cyclocarpum Griseb. } \\
\text { Piich }\end{array}$ & Fabaceae & saprobio \\
\hline & & & & $\begin{array}{l}\text { Bursera simaruba (L.) Sarg. } \\
\text { Chakaa' }\end{array}$ & Burseraceae & \\
\hline & & & & $\begin{array}{l}\text { Brosimum alicastrum Sw. } \\
\text { Ramón }\end{array}$ & Moraceae & \\
\hline $\begin{array}{l}\text { Hypoxylon sublimbatum } \\
\text { (Durieu \& Mont.) P. M. D. } \\
\text { Martín }\end{array}$ & & $x$ & & $\begin{array}{l}\text { Senna racemosa (Mill.) H.S. Irwin } \\
\text { \& Barneby } \\
\text { Ja' abin }\end{array}$ & Fabaceae & saprobio \\
\hline
\end{tabular}


Cuadro 1: Continuación.

\begin{tabular}{|c|c|c|c|c|c|c|}
\hline Especie & $\begin{array}{l}\text { Centro de } \\
\text { Conservación } \\
\text { y Educación } \\
\text { Ambiental }\end{array}$ & $\begin{array}{l}\text { Parque } \\
\text { Ecológico } \\
\text { Punta Sur }\end{array}$ & $\begin{array}{l}\text { Zona Arqueológica } \\
\text { San Gervasio, } \\
\text { Chen-tuk }\end{array}$ & $\begin{array}{l}\text { Hospedero: } \\
\text { Nombre científico } \\
\text { Nombre común }\end{array}$ & Familia & Hábito \\
\hline $\begin{array}{l}\text { Jackrogersella multiformis } \\
\text { (Fr.) L. Wendt, Kuhnert \& } \\
\text { M. Stadler }\end{array}$ & & & $x$ & $\begin{array}{l}\text { Rhizophora mangle } \mathrm{L} \text {. } \\
\text { Mangle rojo }\end{array}$ & Rhizophoraceae & saprobio \\
\hline $\begin{array}{l}\text { Kretzschmaria zonata (Lév.) } \\
\text { P.M.D. Martin }\end{array}$ & & & $x$ & Cedrela odorata L. Cedro & Meliaceae & fitopatógeno \\
\hline $\begin{array}{l}\text { Lasiodiplodia theobromae } \\
\text { (Pat.) Griffon \& Maubl. }\end{array}$ & & $x$ & & $\begin{array}{l}\text { Carica papaya } \mathrm{L} . \\
\text { Papaya }\end{array}$ & Caricaceae & fitopatógeno \\
\hline $\begin{array}{l}\text { Macrovalsaria megalospora } \\
\text { (Mont.) Sivan. }\end{array}$ & $x$ & & & $\begin{array}{l}\text { Pithecellobium dulce (Roxb.) } \\
\text { Benth. }\end{array}$ & Fabaceae & fitopatógeno \\
\hline Patellaria atrata (Hedw.) Fr. & & $x$ & & $\begin{array}{l}\text { Rhizophora mangle L. } \\
\text { Mangle rojo }\end{array}$ & Rhizophoraceae & saprobio \\
\hline $\begin{array}{l}\text { Peroneutypa scoparia } \\
\text { (Schwein.) Carmarán \& A.I. } \\
\text { Romero }\end{array}$ & & $x$ & & $\begin{array}{l}\text { Enterolobium cyclocarpum Griseb. } \\
\text { Piich }\end{array}$ & Fabaceae & saprobio \\
\hline $\begin{array}{l}\text { Phillipsia domingensis (Berk.) } \\
\text { Berk. ex Denison }\end{array}$ & $x$ & & $x$ & $\begin{array}{l}\text { Sideroxylon foetidissimum Jacq. } \\
\text { Caracolillo }\end{array}$ & Sapotaceae & saprobio \\
\hline $\begin{array}{l}\text { Platystomum mexicanum } \\
\text { Raymundo, Martínez- } \\
\text { Pineda, A. Tun \& R. Valenz. }\end{array}$ & & $x$ & & $\begin{array}{l}\text { Manilkara zapota (L.) P. Royen } \\
\text { Sapotillo }\end{array}$ & Sapotaceae & saprobio \\
\hline $\begin{array}{l}\text { Rhytidhysteron neorufulum } \\
\text { Thambug. \& K.D. Hyde }\end{array}$ & $x$ & $x$ & $x$ & $\begin{array}{l}\text { Plumeria obtusa } \mathrm{L} . \\
\text { Flor de mayo }\end{array}$ & Apocynaceae & saprobio \\
\hline $\begin{array}{l}\text { Rhytidhysteron rufulum } \\
\text { (Spreng.) Speg. }\end{array}$ & $x$ & $x$ & $x$ & $\begin{array}{l}\text { Sideroxylon foetidissimum Jacq. } \\
\text { Caracolillo }\end{array}$ & Sapotaceae & saprobio \\
\hline $\begin{array}{l}\text { Rosellinia subiculata (Durieu } \\
\text { \& Mont.) Pass. }\end{array}$ & & & $x$ & $\begin{array}{l}\text { Bursera simaruba (L.) Sarg. } \\
\text { Chakaa' }\end{array}$ & Burseraceae & saprobio \\
\hline $\begin{array}{l}\text { Unguiculariopsis ravenelli } \\
\text { (Berk. \& M.A. Curtis) W.Y. } \\
\text { Zhuang \& Korf }\end{array}$ & & & $x$ & $\begin{array}{l}\text { Rhytidhysteron rufulum (Spreng.) } \\
\text { Speg. }\end{array}$ & Hysteriaceae & mycoparasito \\
\hline Xylaria bambooensis Lloyd & & & $x$ & $\begin{array}{l}\text { Bambusa vulgaris Schrad. ex J.C. } \\
\text { Wendl } \\
\text { Bambú }\end{array}$ & Poaceae & saprobio \\
\hline Xylaria cubensis (Mont.) Fr. & & & $x$ & $\begin{array}{l}\text { Sideroxylon foetidissimum } \\
\text { Caracolillo }\end{array}$ & Sapotaceae & saprobio \\
\hline Xylaria mellisii (Berk.) Cooke & $x$ & & & $\begin{array}{l}\text { Delonix regia (Bojer ex Hook.) Raf. } \\
\text { Flamboyán }\end{array}$ & Fabaceae & saprobio \\
\hline
\end{tabular}

Hypoxylon erytrostroma, H. haematostroma, H. lenormandii, rickii y Kretzschmaria zonata sobre Cedrela odorata. El resto H. subgilvum y Rosellinia subiculosa sobre Bursera simaruba. de las especies de ascomicetos y hospederos se especifica Hypoxylon cinnabarinum, H. haematostroma, H. jaklitschii, en el Cuadro 1.

$H$. lenormandii, $H$. perforatum, $H$. rickii y $H$. subgilvum en

Por otro lado, Hypoxylon haematostroma e H. lenorBrosmium alicastrum. Bertia tropicalis, Cookeina tricholoma, mandii (Hypoxylaceae) se encontraron sobre un mayor núHypoxylon haematostroma, H. investiens, H. lenormandii, H. $\quad$ mero de hospederos: Brosimum alicastrum, Bursera sima- 
ruba, Cedrela odorata, Enterolobium cyclocarpum y Delonix regia (Bojer ex Hook.) Raf. en la primera especie y los cuatro primeros en la segunda. Asimismo, Annulohypoxylon nitens crece sobre Guazuma ulmifolia Lam., Mimosa bahamensis Benth. y Senna racemosa (Mill.) H.S. Irwin \& Barneby e $H$. subgilvum sobre Brosimum alicastrum, Bursera simaruba y Enterolobium cyclocarpum. Solo dos especies presentaron especificidad de hospederos: Xylaria bambooensis sobre Bambusa vulgaris Schrad. ex J.C. Wendl. y Unguiculariopsis ravenelli sobre Rhytidhysteron rufulum, siendo está ultima la única que crece como fungícola.

En Quintana Roo se han registrado 122 especies de ascomicetos, considerando los trabajos de Kohlmeyer (1984), González et al. (2001), Barbosa-Reséndiz et al. (2020), Cobos-Villagrán et al. (2020), De la Fuente et al. (2020), Reyes et al. (2020) y García-Martínez et al. (2021a, b). Asímismo, de las 122 mencionadas para el estado, se han citado 43 taxa en la Reserva de la Biosfera Isla Cozumel (Kohlmeyer, 1984; González et al., 2001; Barbosa-Reséndiz et al., 2020; CobosVillagrán et al., 2020; Reyes et al., 2020 y García-Martínez et al., 2021a, b). Por otro lado, si consideramos que en el presente trabajo se determinaron 40 hongos del phylum Ascomycota, de los cuales 15 son nuevos registros para Quintana Roo, uno es nuevo registro para México (Bertia tropicalis) y una especie nueva (Platystomum mexicanum) y sumamos estos a las 122 especies del estado, se tendrían 139 especies para la entidad. Asimismo, si añadimos a los 43 taxones de la reserva estos 17 reportes nuevos, adicionando ocho que se citan por primera vez para la isla, aumentaríamos a 68 los registros para el municipio Cozumel.

\section{Conclusiones}

De las 40 especies estudiadas se aportaron 17 nuevos registros para Quintana Roo incluidos una nueva especie, Platystomum mexicanum, y un nuevo registro para el país, Bertia tropicalis. De estas 40, 25 fueron mencionados por primera vez para la Reserva de la Biosfera Isla Cozumel. Se incrementó a 139 el conocimiento de los ascomicetos para la entidad y la reserva, con 68 taxa, tiene $48.9 \%$ del total. El Área Natural Protegida con más especies fue la Zona Arqueológica de San Gervasio con 25, siguiéndole el Centro de Conservación y Educación Ambiental con 23 y el Parque Ecológico Punta Sur con 11. De las cuatro clases del phylum Ascomycota, los Sordariomycetes fueron los más representados con 28 taxones, siguiéndole Dothideomycetes con nueve, Pezizomycetes con dos y Leotiomycetes con uno. Los grupos taxonómicos más diversos fueron el orden Xylariales (25 spp.), la familia Hypoxylaceae (16 spp.) y el género Hypoxylon (11 spp.). Se encontraron con mayor frecuencia Rhytidhysteron rufulum (14 especímenes) y Annulohypoxylon nitens (13). Los taxones presentes en todas las localidades fueron Bionectria ochroleuca, Hypoxylon lenormandii, Rhytidhysteron neorufulum y $R$. rufulum. Los ascomicetos de la Isla Cozumel crecen principalmente como saprobios y fitopatógenos. Sin embargo, es necesario continuar con los estudios para establecer los nichos ecológicos de estos organismos y sus relaciones con las plantas en este ecosistema. Este estudio representa una primera aproximación a la riqueza taxonómica de hongos en la isla, por lo que se requiere ampliar el número de exploraciones para un mejor conocimiento de la diversidad fúngica en el caribe mexicano.

\section{Contribución de autores}

TR y RV concibieron y diseñaron el estudio. Todos los autores realizaron las recolecciones y determinaciones de las especies. Las fotos de las figuras fueron tomadas por RV y TR. TR redactó el manuscrito con la ayuda de MMP, AAT, PER, ACV y YAGM. Todos los autores contribuyeron a la discusión, revisión y aprobación del manuscrito final.

\section{Financiamiento}

Este estudio fue apoyado por el Instituto Politécnico Nacional a través de la Secretaría de Investigación y Posgrado (SIP), en los siguientes proyectos: 20180243, 20180244, 20195221, 20195222, 20200248, 20200956, 20210315, 20210661. Los autores agradecen al Consejo Nacional de Ciencia y Tecnología por el financiamento al proyecto 252934 de la convocatoria Ciencia Básica Secretaría de Educacion Pública - Consejo Nacional de Ciencia y Tecnología 2015. PER, ACV, AYGM fueron becarias del Consejo Nacional de Ciencia y Tecnología para realizar sus estudios de Maestría en Biociencias en la Escuela Nacional de Ciencias Biológicas del Instituto Politécnico Nacional y contaron con la beca de Estímulo Institucional de Formación de Investigadores (BEIFI). RV y TR recibieron la beca de la Comisión 
de Operación y Fomento de las Actividades Académicas del IPN, la beca Estímulo al Desempeño a la Investigación de la Secretaria de Posgrado e Investigación del IPN, y la del Sistema Nacional de Investigadores, para realizar sus investigaciones.

\section{Agradecimientos}

Los autores agradecen a las autoridades de la Escuela Nacional de Ciencias Biológicas (ENCB) del Instituto Politécnico Nacional, las facilidades otorgadas para realizar el presente estudio. A Emilio Villanueva Sosa, director de la Fundación Parques y Museos de Cozumel, a Antonio H. Cruz Poot, director del parador turístico de San Gervasio, a José de Jesús Benavides Andrade, director del parque Ecoturístico Punta Sur y a Lorena Nohemi Flores Morcillo, asistente de dirección. Se reconoce el apoyo de Alfonso Daniel Gay González en la elaboración del mapa.

\section{Literatura citada}

Álvarez, V. I., T. Raymundo y R. Valenzuela. 2016. Hongos histerioides (Dothideomycetes, Ascomycota) del Bosque Tropical Caducifolio en el Parque Nacional Lagunas de Chacahua, Oaxaca, México. Acta Botanica Mexicana 116: 49-64. DOI: https:// doi.org/10.21829/abm116.2016.1118

Angelini, C. y G. Medardi. 2012. Tropical fungi: twelve species of lignicolous Ascomycota from the Dominican Republic. Mycosphere 3(5): 567-601. DOI: https://doi.org/10.5943/ mycosphere/3/5/6

Angulo, A., J. Rueda-Almonacid, J. Rodríguez-Mahecha y E. La Marca. 2006. Técnicas de inventario y monitoreo para los anfibios de la región tropical andina. Conservación Internacional. Serie Manuales de Campo No. 2. Panamericana Formas e Impresos S.A. Bogotá, D.C., Colombia. Pp. 135-137.

Ávalos-Lázaro, A., J. Rosique, S. Cappello y J. L. Villarruel. 2018. Ascomicetes (Fungi: Ascomycota) del Parque Estatal Agua Blanca, Macuspana, Tabasco, México. Acta Botanica Mexicana 122: 141-154. DOI: https://doi.org/10.21829/abm122.2018.1261

Barbosa-Reséndiz, A., R. Valenzuela, M. Sánchez-Flores, S. Bautista-Hernández, A. Cobos-Villagrán, A. Pérez-Valdespino, R. Espinoza-Mellado, M. Martínez-Pineda y T. Raymundo. 2020. El género Daldinia (Sordariomycetes, Ascomycota) en México. Acta Botanica Mexicana 127: e1600. DOI: https://doi. org/10.21829/abm127.2020.1600
Becerril-Navarrete, A. M., V. M. Goméz-Reyes, E. N. Palestina Villa y R. Medel-Ortiz. 2018. Nuevos registros de Xylaria (Xylariaceae) para el estado de Michoacán, México. Scientia Fungorum 48: 61-75. DOI: https://doi.org/10.33885/ sf.2018.48.1199

Carmarán, C. C., A. I. Romero y L. M. Giussani. 2006. An approach towards a new phylogenetic classification in Diatrypaceae. Fungal Diversity 23: 67-87.

Chacón, S. 2003. Estudio monográfico de los Diatrypales (Ascomycotina) en México. Tesis doctoral. Facultad de Ciencias, Universidad Nacional Autónoma de México. México, D.F., México. 331pp.

Chacón, S. y F. Tapia. 2016. Algunas especies saprobias de Dothideomycetes y Lecanoromycetes (Pezizomycotina: Ascomycota) en México. Revista Mexicana de Biodiversidad 87(4): 1169-1176. DOI: http://doi.org/10.1016/j.rmb.2016.10.013

Chacón, S., F. Tapia y M. Esqueda. 2014. New records of Dothideomycetes from Mexico. Mycotaxon 128: 145-157. DOI: https:// doi.org/10.5248/128.145

Chaverri, P., S. Huhndorf, J. D. Rogers y G. J. Samuels. 2011a. Microhongos comunes de Costa Rica y otras regiones tropicales. Instituto Nacional de Biodiversidad. Santo Domingo de Heredia, Costa Rica. 241 pp.

Chaverri, P., S. Salgado, Y. Hirooka, A. Y. Rossman y G. J. Samuels. 2011b. Delimitation of Neonectria and Cylindrocarpon (Nectriaceae, Hypocreales, Ascomycota) and related genera with Cylindrocarpon like anamorphs. Studies in Mycology 68: 5778. DOI: https://doi.org/10.3114/sim.2011.68.03

Cibrián-Tovar, D. 2013. Manual para la identificación y manejo de plagas en plantaciones forestales comerciales. Comisión $\mathrm{Na}$ cional Forestal - Secretaría de Medio Ambiente de Recursos Naturales, México, Universidad Autónoma Chapingo. Texcoco, México. 229 pp.

Cibrián-Tovar, D., D. Alvarado-Rosales y S. E. García-Díaz. 2007. Enfermedades forestales en México/Forest diseases in Mexico. Universidad Autónoma de Chapingo; Comisión Nacional Forestal-Secretaría de Medio Ambiente y Recursos Naturales, México; Forest Service, Uniated States Department of Agriculture, EUA; Canadian Forest Service, Natural Resources Canada; Comisión Forestal de América del Norte, Food and Agriculture Organization. Texcoco, México. 587 pp.

Cobos-Villagrán, A., C. H. Hernández-Rodríguez, R. Valenzuela, L. Villa-Tanaca, R. P. Calvillo-Medina, L. E. Mateo-Cid, M. Mar- 
tínez-Pineda y T. Raymundo. 2020. El género Rhytidhysteron (Dothideomycetes, Ascomycota) en México. Acta Botanica Mexicana 127: e1675. DOI: https://doi.org/10.21829/ abm127.2020.1675

CONANP. 2007. Estudio Previo Justificativo para el establecimiento del Área de Protección de Flora y Fauna Isla de Cozumel, Quintana Roo, México. Comisión Nacional de Áreas Naturales Protegidas-Secetaría de Medio Ambiente y Recursos Naturales. México. 126 pp.

CONANP. 2016. Programa de Manejo Área de Protección de Flora y Fauna la porción norte y la franja costera oriental, terrestres y marinas de la Isla de Cozumel. Comisión Nacional de Áreas Naturales Protegidas-Secetaría de Medio Ambiente y Recursos Naturales. Iztapalapa, D.F., México. 243 pp.

De la Fuente, J. L., J. García-Jiménez, C. Y. López, I. Oros-Ortega, R. Y. Vela-Hernández, G. Guevara-Guerrero, F. Garza-Ocañas, J. A. Casanova, L. E. Ibarra-Garibay y V. M. Bandala-Muñoz. 2020. An annotated checklist of the macrofungi (Ascomycota, Basidiomycota and Glomeromycota) from Quintana Roo, Mexico. Check List 16 (3): 627-648. DOI: https://doi. org/10.15560/16.3.627

Denison, W. C. 1963. A preliminary study of the operculate cup-fungi of Costa Rica. Revista Biologia Tropical 11: 99-129.

Fournier, J., C. Lechat C. y R. Courtecuisse. 2015. The genus Hypoxylon (Xylariaceae) in Guadeloupe and Martinique (French West Indies). Ascomycete.org 7(5): 145-212.

García, E. 2004. Modificaciones al sistema de clasificación climática de Koppen, Serie Libros No. 6. Instituto de Geografía, Universidad Nacional Autónoma de México. México, D.F., México. 90 pp.

García-Martínez, Y. A., G. Heredia, R. Valenzuela and T. Raymundo. 2021a. First record of Mycoporum buckii (Dothideomycetes, Pleosporales) in Mexico. Acta Botanica Mexicana 128: e1802. DOI: https://doi.org/10.21829/abm128.2021.1802

García-Martínez, Y. A., G. Heredia Abarca, J. Guzmán-Guillermo, R. Valenzuela y T. Raymundo. 2021b. Hongos asociados al mangle rojo Rhizophora mangle (Rhizophoraceae) en la Reserva de la Biosfera Isla Cozumel, Quintana Roo, México. Acta Botanica Mexicana 128: e1792. DOI: https://doi.org/10.21289/ abm128.2021.1792

González, M. C., R. T. Hanlin y M Ulloa. 2001. A checklist of higher marine fungi of Mexico. Mycotaxon 80: 241-253.
Guzmán, G. 1983. Los hongos de la península de Yucatán II. Nuevas exploraciones y adiciones micológicas. Biotica 8: 71-87.

Guzmán, G. 2003. Los hongos de El Edén Quintana Roo (introducción a la micobiota tropical de México). Instituto de Ecología A. C., Comisión Nacional para el Conocimiento y Uso de la Biodiversidad. Xalapa, México. 316 pp.

Guzmán, G. y D. A. García Saucedo. 1973. Macromicetos del estado de Jalisco. I. Consideraciones generales y distribución de las especies conocidas. Boletín de la Socieda Mexicana de Micología 7: 129-143.

Guzmán-Dávalos, L. y G. Guzmán. 1979. Estudio ecológico comparativo entre los hongos (macromicetos) de los bosques tropicales y de los de coníferas del sureste de México. Boletín de la Socieda Mexicana de Micología 13: 89-125.

Guzmán-Dávalos, L., G. Nieves y G. Guzmán. 1983. Hongos del estado de Jalisco II. Boletín de la Sociedad Mexicana de Micología 18: 125-158.

Heredia, G. 1989. Estudio de los hongos de la reserva de la Biósfera El Cielo, Tamaulipas. Consideraciones sobre la distribución y ecología de algunas especies. Acta Botanica Mexicana 7: 1-18. DOI: https://doi.org/10.21829/abm7.1989.577

Hladki, A. I. y A. I. Romero. 2009. Novedades para los géneros Annulohypoxylon e Hypoxylon (Ascomycota, Xylariaceae) en la República Argentina. Darwiniana 47(2): 278-288.

Huhndorf, S. M., A. N. Miller y F. A. Fernández. 2004. Molecular systematics of the Coronophorales and new species of Bertia, Lasiobertia and Nitschkia. Mycological Research 108(12): 13841398. DOI: https://doi.org/10.1017/s0953756204001273

Hyde, K. D. y S. Lee. 1995. Ecology of mangrove fungi and their roles in nutrients cycling: What gaps occur our Knowledge. Hydrobiologia 295: 107-118. DOI: https://doi.org/10.1007/ BF00029117

Ju, Y. M. y J. D. Rogers. 1996. A revision of the genus Hypoxylon. Mycologia Memoir No. 20. APS Press. Saint Paul, Minesota, USA. 365 pp.

Kirk, P. M., P. F. Cannon, D. W. Minter y J. A. Stalpers. 2008. Ainsworth and Bisby's Dictionary of the fungi. 10th ed. CAB International. Wallingford, UK. 771 pp.

Kohlmeyer, J. 1984. Tropical marine fungi. Marine Ecology 5(4): 329378. DOI: https://doi.org/10.1111/j.1439-0485.1984.tb00130.x

Martin, P. 1969. Studies in the Xylariaceae V: Euhypoxylon. Journal of South African Botany 35: 149-206. 
Medel, R. 2002. Nuevos registros de Pyrenomycetes (Ascomycotina) en México. Boletín de la Sociedad Botánica de México 70: 79-85. DOI: https://doi.org/10.17129/botsci.1656

Medel, R. y S. Chacón. 1988. Ascomycetes lignícolas de México, II. Algunos Pyrenomycetes y Discomycetes. Micología Neotropical Aplicada 1: 87-96.

Medel, R., R. Castillo y G. Guzmán. 2008. Las especies de Xylaria (Ascomycota, Xylariaceae) conocidas de Veracruz, México y discusión de nuevos registros. Revista Mexicana de Micología 28: 101-118.

Méndez-Mayboca, F., J. Checa, M. Esqueda y S. Chacón. 2010. New records of Loculoascomycetes from natural protected areas in Sonora, Mexico. Mycotaxon 111(1): 19-30. DOI: https://doi.org/10.5248/111.19

Mugambi, G. K. y S. M. Huhndorf. 2010. Multigene phylogeny of the Coronophorales: morphology and new species in the order. Mycologia 102(1): 185-210. DOI: https://doi. org/10.3852/09-043

Ortega-López, I., R. Valenzuela, A. D. Gay-González, B. N. LaraChavez, E. O. López-Villegas y T. Raymundo. 2019. La Familia Sarcoscyphaceae (Pezizales, Ascomycota) en México. Acta Botanica Mexicana 126: e1430. DOI: https://doi. org/10.21829/abm126.2019.1430

Palacios-Mayorga, S., A. L. Anaya, E. González-Velazquez, L. Huerta-Arcos y A. Gomez-Pompa. 2003. Periphyton as a potencial biofertilizer in intensive agriculture of the ancient Maya. In: Gómez-Pompa, A., M. Allen, S. L. Fedick y J. J. JiménezOsornio (eds.). The lowland maya area: three millennia at the human-wildland interface. Food Products Press. Nueva York, USA. Pp. 389-400.

Pompa-González, A., E. Aguirre-Acosta, A. V. Encalada-Olivas, A. De Anda-Jáuregui, J. Cifuentes-Blanco y R. Valenzuela-Garza. 2011. Los Macromicetos del Jardín Botánico de ECOSUR “Dr. Alfredo Barrera Marín” Puerto Morelos, Quintana Roo. Colección Corredor Biológico Mesoamericano México, Serie Diálogos No. 6. Comision Nacional de Biodiversidad. México, D.F., México. 108 pp.

Portugal, D., E. Montiel, L. López y V. M. Mora. 1985. Contribución al conocimiento de los hongos que crecen en la región del Texcal, estado de Morelos. Revista Mexicana de Micología 1: 401-412.

Raymundo, T., M. Coronado, A. Gutiérrez, M. Esqueda y R. Valenzuela. 2017a. New records of Ascomycota from tropical dry forest in Sonora, Mexico. Mycotaxon 132(2): 421-432. DOI: https://doi.org/10.5248/132.421

Raymundo, T., M. Martínez-Pineda, A. Cobos-Villagrán, M. Sánchez-Flores y R. Valenzuela. 2020. Primer registro de Unguiculariopsis ravenelii (Leotiomycetes, Ascomycota) en México. Acta Botanica Mexicana 127: e1666. DOI: https://doi. org/10.21829/abm127.2020.1666

Raymundo, T., R. Soto-Agudelo, S. Bautista-Hernández, A. R. Morales-Campos y R. Valenzuela. 2016. Catálogo de los ascomicetos del bosque mesófilo de montaña de Tlanchinol, Hidalgo. Boletín de la Sociedad Micológica de Madrid 40: 83-104.

Raymundo, T., E. Escudero, I. Ortega, D. Castro, H. León y R. Valenzuela. 2014. Ascomicetos del bosque tropical caducifolio en el Parque Nacional Lagunas de Chacahua, Oaxaca, México. Boletín de la Sociedad Micológica de Madrid 38: 9-21.

Raymundo, T., E. Escudero-Leyva, R. Soto-Agudelo, J. García-Jiménez, L. Romero-Bautista y R. Valenzuela. 2017b. Nuevos registros de Hypocreales (Sordariomycetes, Ascomycota) del bosque mesófilo de montaña de la Sierra Alta Hidalguense en México. Acta Botanica Mexicana 120: 39-57. DOI: https://doi.org/10.21829/abm120.2017.1263

Reyes, P. E., R. Valenzuela y T. Raymundo. 2020. El género Hypoxylon (Xylariales, Ascomycota) en el bosque tropical caducifolio de cuatro Áreas Naturales Protegidas de México. Acta Botanica Mexicana 127: e1680. DOI: https://doi. org/10.21829/abm127.2020.1680

Robles-Porras, L., M. Ishiki-Ishihara y R. Valenzuela. 2006. Inventario preliminar de los Macromicetos en los Altos de Chiapas, México. Polibotánica 21: 89-101.

Rogers. J. D. 1985. Hypoxylon duranii sp. nov. and the anamorphs of $H$. caries, $H$. papillatum, and Rosellinia subiculata. Mycotaxon 23: 429-437.

Romero-Nájera, I. 2004. Distribución, abundancia y uso de habitat de Boa constrictor introducida a la Isla Cozumel. Tesis de maestría. Centro de Investigaciones en Ecosistemas, Universidad Nacional Autónoma de Mexico. Morelia, México. 409 pp.

Sánchez-Jácome, M. R. y L. Guzmán-Dávalos. 2011. Hongos citados para Jalisco, II. Ibugana 16: 25-60.

San Martín, F. y P. A. Lavin. 1997. Datos sobre los géneros Entonaema y Ustulina (Pyrenomycetes, Xylariaceae). Acta Botanica Mexicana 40: 25-35. DOI: https://doi.org/10.21829/ abm40.1997.780 
San Martín, F. y J. D. Rogers. 1989. A preliminary account of Xylaria of Mexico. Mycotaxon 34: 283-374.

San Martín, F. y J. D. Rogers. 1993. Biscogniauxia and Camillea in Mexico. Mycotaxon 47: 229-258.

San Martín, F. y J. D. Rogers. 1995. Rosellinia and Thamnomyces in Mexico. Mycotaxon 53: 115-127.

San Martín, F. y J. D. Rogers. 1996. A preliminary list of Xylariaceous fungi at El Cielo Biosphere reserve in Tamaulipas, Mexico. Acta Botanica Mexicana 37: 23-31. DOI: https:// doi.org/10.21829/abm37.1996.766

San Martín, F., Y. M. Ju y J. D. Rogers. 1999. Algunas especies de Hypoxylon (Pyrenomycetes, Xylariaceae) de México. Acta Botanica Mexicana 47: 31-53. DOI: https://doi.org/10.21829/ abm47.1999.823

Thambugala, K. M., K. D. Hyde, P. D. Eungwanichayapant, A. I. Romero y Z. Y. Liu. 2016. Additions to the genus Rhytidhysteron in Hysteriaceae. Cryptogamie Mycologie 37(1): 99-116. DOI: https://doi.org/10.7872/crym/v37.iss1.2016.99

Ulloa, M. y R. T. Hanlin. 2006. Nuevo diccionario ilustrado de Micología. APS Press. St. Paul, USA. 615 pp.

Valencia-Botín, A. J., J. S. Sandoval-Islas, E. Cárdenas-Soriano, T. J. Michailides y G. Rendón-Sánchez. 2003. Botryosphae- ria dothidea causing stem spots on Hylocereus undatus in Mexico. Plant Payhology 52(6): 803. DOI: https://doi. org/10.1111/j.1365-3059.2003.00912.x

Vassiljeva, L. N., H. X. Ma, A. V. Chernyshev y S. L. Stephenson. 2015. Bertia hainanensis sp. nov. (Coronophorales) from southern China. Mycotaxon 130(1): 197-205. DOI: http:// doi.org/10.5248/130.197

Webster, J. y R. W. S. Weber. 2007. Introduction to Fungi. 3a ed. Cambridge University Press. New York, USA. 819 pp.

Welden, A. L. y G. Guzmán. 1978. Lista preliminar de los hongos, líquenes y mixomicetos de las regiones de Uxpanapa, Coatzacoalcos, Los Tuxtlas, Papaloapan y Xalapa (parte de los estados de Veracruz y Oaxaca). Boletín de la Sociedad Mexicana de Micología 26: 1-24.

Wendt, L., E. B. Sir, E. Kuhnert, S. Heitkämper, C. Lambert, A. I. Hladki, A. I. Romero, J. J. Luangsa-ard, P. Srikitikulchai, D. Peršoh y M. Stadler. 2018. Resurrection and emendation of the Hypoxylaceae, recognised from a multigene phylogeny of the Xylariales. Mycological Progress 17: 115-154. DOI: https://doi.org/10.1007/s11557-017-1311-3 